\title{
Blocking mitochondrial calcium release in Schwann cells prevents demyelinating neuropathies
}

\author{
Sergio Gonzalez, ${ }^{1}$ Jade Berthelot, ${ }^{1}$ Jennifer Jiner, ${ }^{1}$ Claire Perrin-Tricaud, ${ }^{1}$ Ruani Fernando, ${ }^{1}$ Roman Chrast, ${ }^{2}$ \\ Guy Lenaers, ${ }^{1,3}$ and Nicolas Tricaud ${ }^{1}$
}

IInstitute for Neurosciences of Montpellier, INSERM U1051, Université de Montpellier, Montpellier, France. ²Departments of Clinical Neuroscience and Neuroscience, Karolinska Institutet, Stockholm, Sweden. ${ }^{3}$ Mitochondrial Medicine Research Centre, Pôle de Recherche et d'Enseignement en Médecine Mitochondriale, Université d'Angers, Angers, France.

\begin{abstract}
Schwann cells produce myelin sheath around peripheral nerve axons. Myelination is critical for rapid propagation of action potentials, as illustrated by the large number of acquired and hereditary peripheral neuropathies, such as diabetic neuropathy or Charcot-Marie-Tooth diseases, that are commonly associated with a process of demyelination. However, the early molecular events that trigger the demyelination program in these diseases remain unknown. Here, we used virally delivered fluorescent probes and in vivo time-lapse imaging in a mouse model of demyelination to investigate the underlying mechanisms of the demyelination process. We demonstrated that mitochondrial calcium released by voltage-dependent anion channel 1 (VDAC1) after sciatic nerve injury triggers Schwann cell demyelination via ERK1/2, p38, JNK, and c-JUN activation. In diabetic mice, VDAC1 activity was altered, resulting in a mitochondrial calcium leak in Schwann cell cytoplasm, thereby priming the cell for demyelination. Moreover, reduction of mitochondrial calcium release, either by shRNA-mediated VDAC1 silencing or pharmacological inhibition, prevented demyelination, leading to nerve conduction and neuromuscular performance recovery in rodent models of diabetic neuropathy and Charcot-Marie-Tooth diseases. Therefore, this study identifies mitochondria as the early key factor in the molecular mechanism of peripheral demyelination and opens a potential opportunity for the treatment of demyelinating peripheral neuropathies.
\end{abstract}

\section{Introduction}

In the peripheral nervous system (PNS), Schwann cells (SCs) are responsible for myelin production, which contributes to axonal protection and allows for efficient action potential transmission $(1,2)$. Unfortunately, acquired and hereditary demyelinating diseases of the PNS are numerous and affect an increasing number of people (3).

Acquired demyelinating diseases are the most common, as they include diabetic peripheral neuropathy $(4,5)$, drug-related peripheral neuropathies, leprosy, and inflammatory neuropathies (6). Diabetic peripheral neuropathy is a major complication of diabetes type 1 and 2 and a cause of considerable morbidity $(7,8)$. Indeed, it has been reported that at least $50 \%$ of diabetic patients develop one or several forms of diabetic neuropathy within 25 years after diagnosis (7). This neuropathy affects both myelinated SCs and peripheral axons/neurons, leading to changes in nerve conduction, and it is often associated with demyelination in the long term (9). However, the molecular mechanisms that lead to these nerve defects remain unclear.

In the PNS, hereditary demyelinating diseases are rare but remain among the most common hereditary diseases (10). While they are rarely lethal, they range from life threatening to severely affecting life and therefore put a high burden on public health systems. Charcot-Marie-Tooth (CMT) diseases, the most common of these diseases, affects about 1 in 2,500 individuals worldwide

Conflict of interest: The authors have declared that no conflict of interest exists. Submitted: August 31, 2015; Accepted: December 18, 2015.

Reference information: J Clin Invest. 2016;126(3):1023-1038. doi:10.1172/JCI84505.
(10). Numerous mutated genes are known to cause demyelinating CMT diseases, and, while the molecular mechanisms of each disease remain unclear, the common result of these mutations is the induction of the SC demyelination program (11-13).

The etiologies of acquired and hereditary peripheral nerve diseases are diverse, but they all result in demyelination and subsequent neuronal death. Thus, an important challenge is to understand the cellular and molecular events that underlie demyelination of SCs. A recent study showed that mitochondrial dysfunctions are involved in axonal degeneration (14). More specifically, mitochondria of the myelin sheath appear to be essential for neuron homeostasis $(15,16)$, but their physiological properties and their role in myelin maintenance remain elusive. In addition, diabetic peripheral neuropathy and some CMT diseases have been linked with perturbations of mitochondrial functions and to mitochondrial stress in the PNS (17). Taken together, this suggests that mitochondria could play an essential role in demyelinating peripheral neuropathies.

In this study, to analyze mitochondrial physiology in myelinating SCs (mSCs) of living mice, we used an approach involving virally delivered mito-targeted fluorescent probes and in vivo time-lapse multiphoton imaging. We discovered that, after induction of SC demyelination, mitochondria release calcium in the cytoplasm before radically changing their physiology. This calcium release is the key event that activates the ERK1/2, p38, JNK, and c-JUN pathways and engages the demyelination program after sciatic nerve injury and in diabetic peripheral neuropathy. We then successfully inhibited this molecular mechanism in diabetic mice and CMT1A rat models, allowing for the maintenance of the myelin sheath and increasing nerve conduction velocity (NCV) and 
A

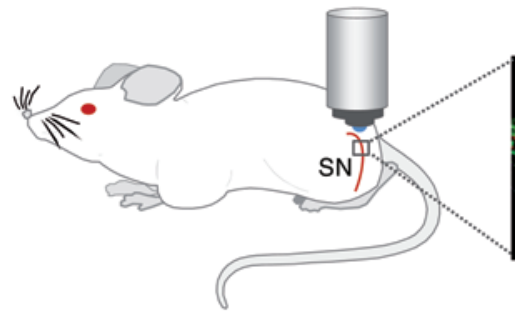

B
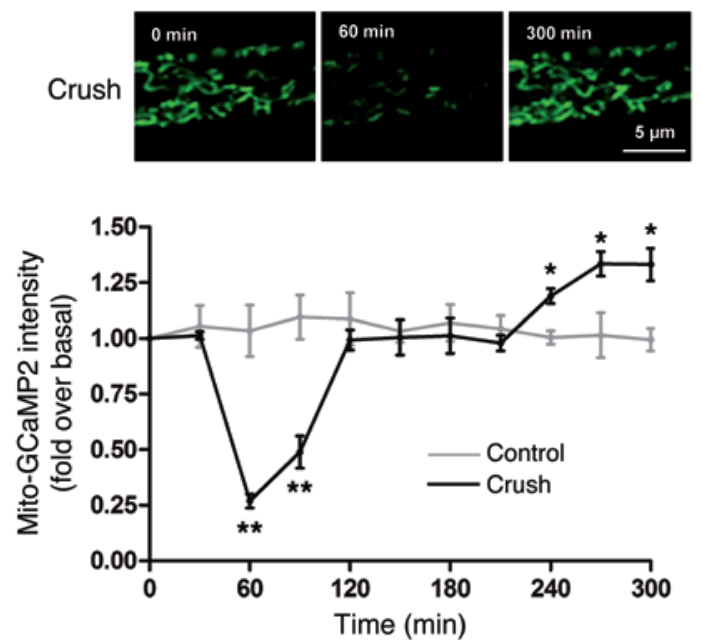

D
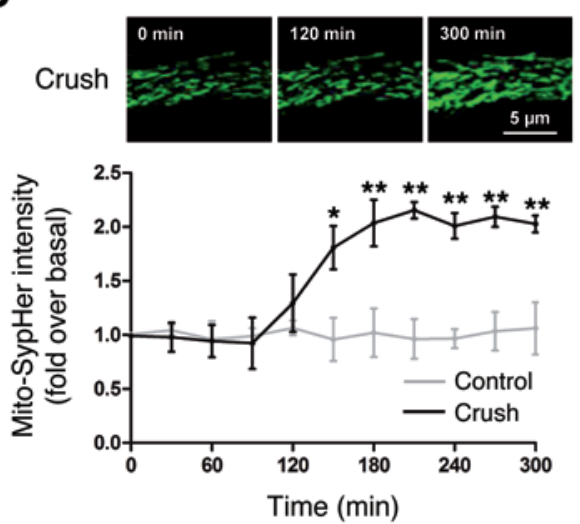

G
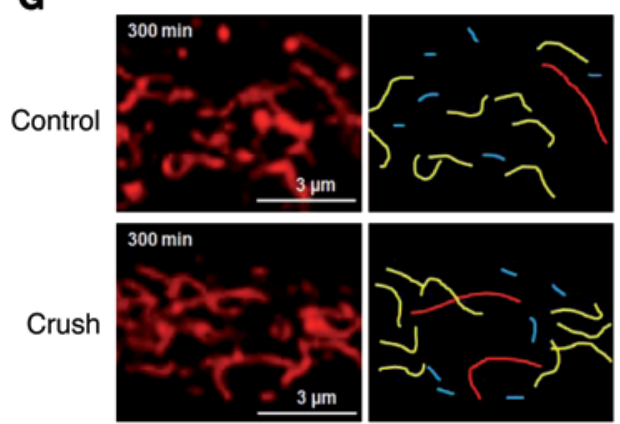

$>3 \mu \mathrm{m}$

미 $>1-3 \mu \mathrm{m}$

- $0.1-1 \mu \mathrm{m}$
E

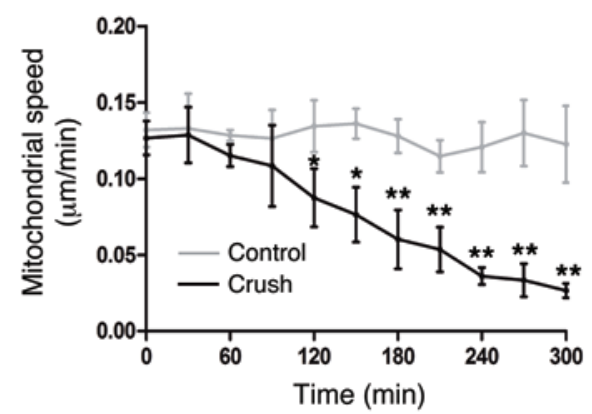

H

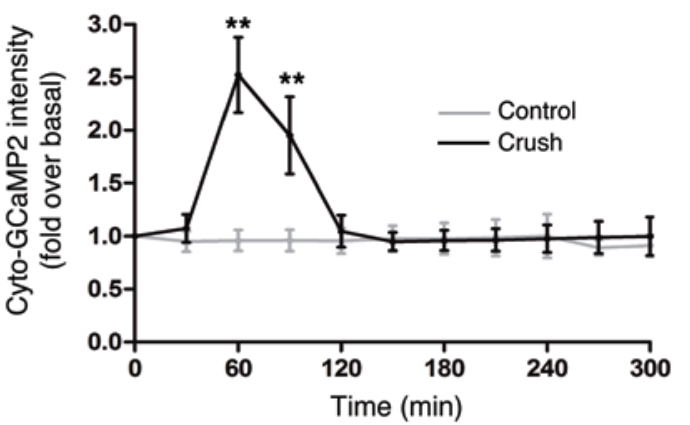

F $\quad \underline{1 h} \underline{2 h} \underline{5 h} \quad \underline{1 h} \underline{2 h} \underline{5 h}$
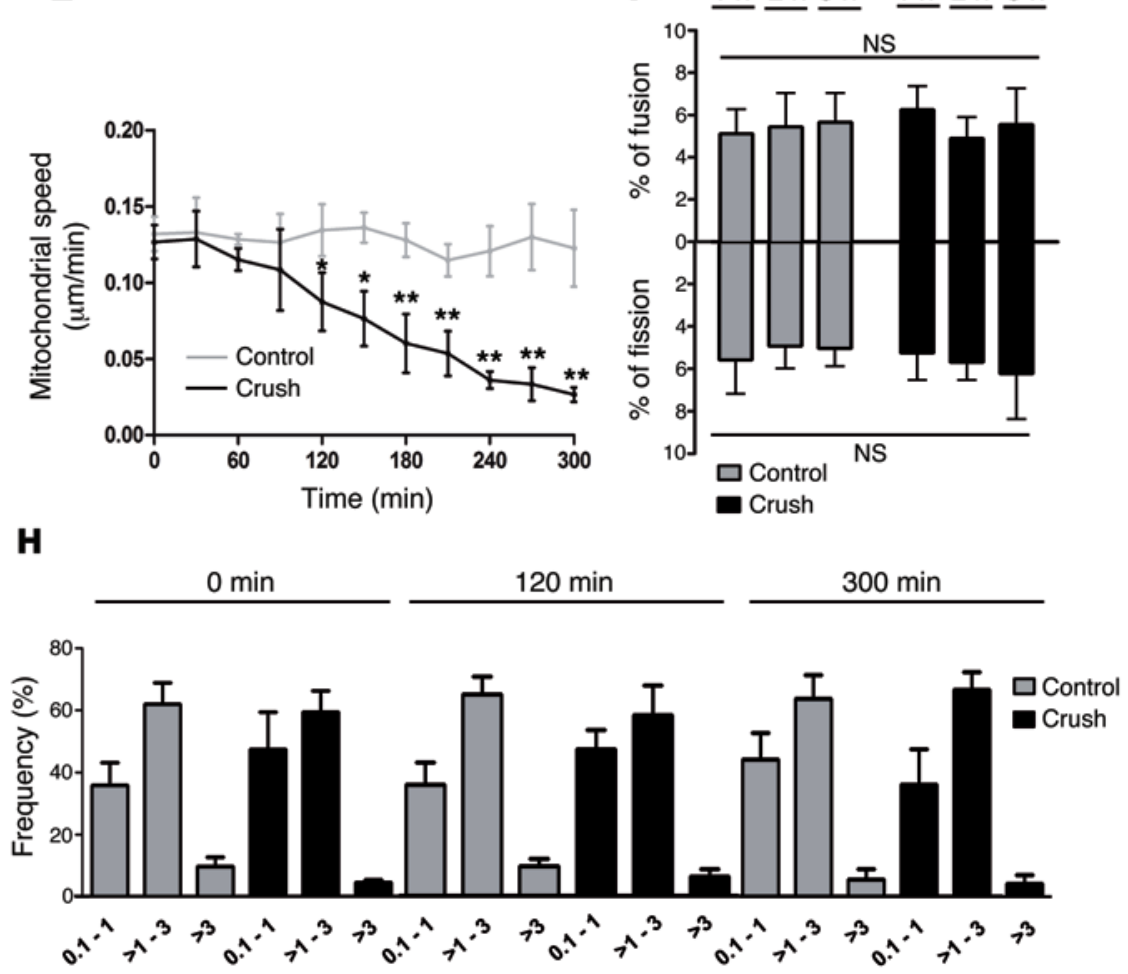

Length $(\mu \mathrm{m})$ 
Figure 1. Mitochondrial physiology changes during SC demyelination. (A) Schematic representation of the imaging technique (left). $\mathrm{mSCs}$ are labeled with $\mathrm{E}$-cadherin (green), mitochondria are labeled with mitodsRed2 (red), and the cell nucleus is labeled with DAPI (blue) (middle). Scale bar: $50 \mu \mathrm{m}$. Representative image of mitochondria imaged with a multiphoton microscope (right). Scale bar: $5 \mu \mathrm{m}$. SN, sciatic nerve. (B) Mitochondrial calcium amount (mito-GCaMP2), (C) cytoplasmic calcium amount (cyto-GCaMP2), (D) mitochondrial pH (mito-SypHer), and (E) mitochondrial motility (mito-dsRed2) in mSCs of control and crushed nerves. Probe intensities are represented as fold over basal conditions (before crush), and motility is represented as $\mu \mathrm{m}$ traveled in 1 minute over 300 minutes of time-lapse acquisition. Representative images of labeled mitochondria. Scale bar: $5 \mu \mathrm{m}$ (B and D); $100 \mu \mathrm{m}$ (C). (F) Ratio of mitochondrial fusion and fission events in control and crushed nerves. (G) Representative images of mSC mitochondria and mitochondrial length quantification in control and crushed nerves after 300 minutes of time-lapse imaging. Scale bar: $3 \mu \mathrm{m}$. (H) Frequency histogram of mitochondrial length in control and crush conditions. No significant difference was found. Data are expressed as the mean \pm SEM. $n=3-6$ mice for each group. Asterisks mark statistical differences compared with noncrushed nerves. ${ }^{*} P<0.05$, ${ }^{* *} P<0.01$, 2-tailed Student's $t$ test.

neuromuscular performance. We describe here an early molecular mechanism that induces SC demyelination, and we show that the inhibition of this mechanism allows for myelin maintenance in rodent models of inherited and acquired peripheral neuropathies.

\section{Results}

Characterization of mitochondrial physiology during demyelination. To characterize mitochondrial physiology during the demyelination process, we combined the nerve crush model to induce demyelination and a viral approach $(18,19)$, allowing the expression of different fluorescent probes selectively into mitochondria of mSCs in mouse sciatic nerves (Figure 1A). Three weeks after virus injection, mice were anesthetized and their sciatic nerves were exposed and placed under a multiphoton microscope, allowing $\mathrm{mSC}$ mitochondria imaging in physiological conditions as described previously (18). Then, the nerve was carefully crushed around $5 \mathrm{~mm}$ upstream of the imaging area and changes occurring in $\mathrm{mSC}$ mitochondria downstream of the crush were recorded. We first expressed the fluorescent probe mitoGCaMP2, which allows real-time mitochondrial calcium recording (ref. 20 and Supplemental Figure 1; supplemental material available online with this article; doi:10.1172/JCI84505DS1). We observed a transitory decrease of mitochondrial calcium starting 1 hour after crush (Figure 1B). We then expressed this probe in the mSC cytoplasm (cyto-GCaMP2), and we observed a transitory increase in cytoplasmic calcium coincident with the loss of mitochondrial calcium (Figure 1C).

We then investigated the mitochondrial $\mathrm{pH}$ using a virus expressing the mitochondria-targeted $\mathrm{pH}$-sensitive probe mitoSypHer (21). Mitochondrial matrix $\mathrm{pH}$ significantly increased after more than 2 hours after nerve injury (Figure 1D), reaching a plateau that was maintained until the end of the experiment. We next investigated mitochondrial dynamics and morphology in demyelinating SCs using the fluorescent protein mito-dsRed2 (18). We observed a progressive decrease of mitochondria motility 1 hour after crush parallel to the mitochondrial calcium release (Figure 1E and Supplemental Videos 1 and 2). This change did not correlate with variation in fusion and fission ratio (Figure $1 \mathrm{~F}$ ) or mitochondrial size (Figure 1, G and $\mathrm{H}$ ), suggesting that the molecular mechanisms allowing mitochondria movements, but not those responsible for fusion and fission events, were altered.

Together, this succession of events suggests that one of the earliest responses of mSCs to nerve injury is a pulse of mitochondrial calcium in the cytoplasm followed by a change in mitochondrial physiology.

VDAC1 is responsible for mitochondrial changes in SCs after nerve crush. Voltage-dependent anion channel 1 (VDAC1) is a porin ion channel located in the outer mitochondrial membrane (22) and strongly expressed in mSC mitochondria (23). VDAC1 is known as a regulator of mitochondrial calcium release, playing a role in cell signaling, apoptosis, and dedifferentiation $(24,25)$. To test whether VDAC1 plays a role in observed mitochondrial calcium release, 2 small inhibitory RNAs effective against mouse VDAC1 expression were selected (Figure 2A) and cloned in viral vectors together with fluorescent probes. These viral vectors were injected in mouse sciatic nerves in order to silence VDAC1 expression in mSCs in vivo. Vectors expressing dsRed2 shRNA (for GCaMP2 and SypHer probes) or GFP shRNA (for the mito-dsRed2 probe) together with fluorescent probes were used as control. Both VDAC1 shRNAs significantly reduced the channel expression in infected cells (Figure 2B). This did not change mitochondrial or cytoplasmic calcium, mitochondrial motility, or $\mathrm{pH}$ in basal conditions (Supplemental Figure 2). However, after nerve crush, shRNA2 significantly reduced and shRNA3 completely inhibited mitochondrial calcium release in mSCs (Figure 2C), showing that VDAC1 mediates the release of mitochondrial calcium. A similar effect was observed when nerves expressing mito-GCaMP2 were treated before crush with TRO19622, a selective ligand of mitochondrial VDAC1 (ref. 26 and Figure 2C), showing that this drug is able to block calcium release from $\mathrm{mSC}$ mitochondria. When nerves were treated with methyl jasmonate (MJ), a drug that increases VDAC permeability (27), mSC mitochondrial calcium concentration sharply decreased 2 hours after injection (Figure $2 \mathrm{G}$ ), showing that VDAC1 opening is sufficient to release mitochondrial calcium, even in absence of crush injury.

We also analyzed cytoplasmic calcium, mitochondrial $\mathrm{pH}$, and mitochondrial motility when cells were silenced for VDAC1 or treated with TRO19622 or MJ. After nerve crush VDAC1 shRNAs and TRO19622 significantly reduced cytoplasmic calcium pulse (Figure 2D), mitochondrial pH increase (Figure 2E), and loss of mitochondrial motility (Figure 2F and Supplemental Video 3), showing that these changes were due to VDAC1 calcium release. In contrast, opening VDAC1 by MJ treatment in absence of crush was sufficient to increase cytoplasmic calcium (Figure 2H) and mitochondrial $\mathrm{pH}$ (Figure 2I) and to reduce mitochondrial motility (Figure 2J).

However, VDAC1 does not span the inner mitochondrial membrane, and any release mechanism also relies on inner membrane channels. The mitochondrial permeability transition pore (mPTP) is a large protein complex that crosses the inner mitochondrial membrane (28) and allows calcium release from the matrix. We hypothesized that MPTP formation was also required for mitochondrial calcium release after nerve injury. To investigate this, we first blocked MPTP formation before crush using cyclosporin A (CsA), a selective MPTP inhibitor (29). The optimal 
A
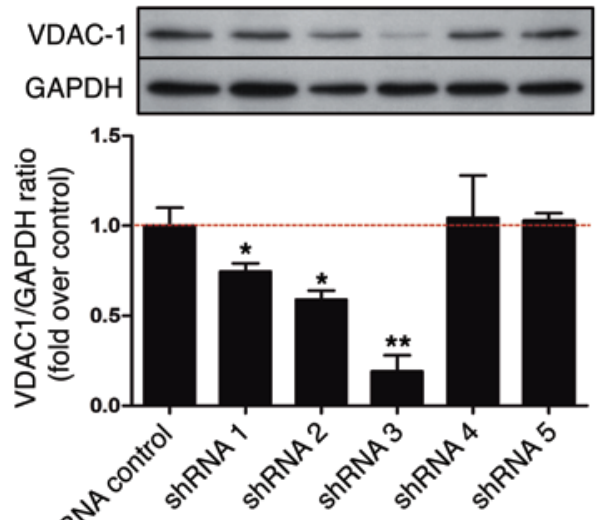

B

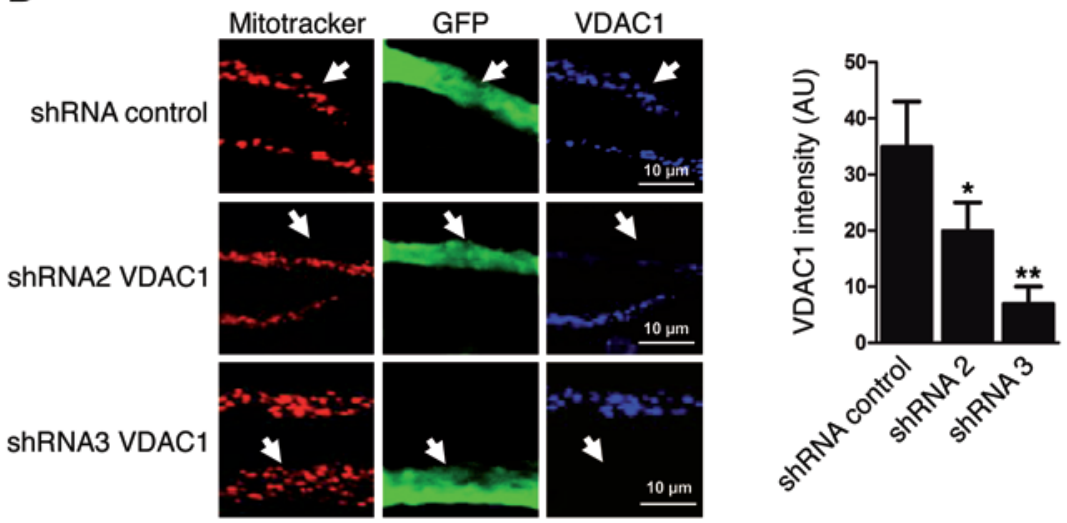

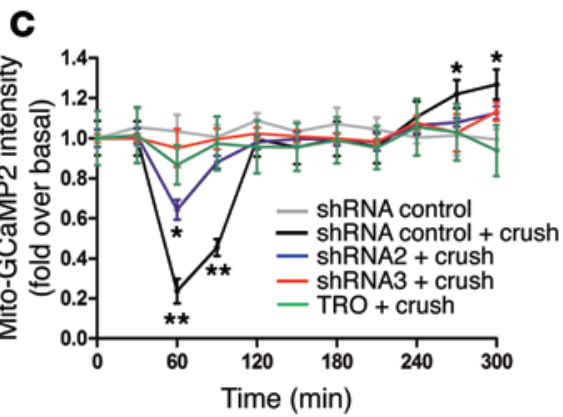

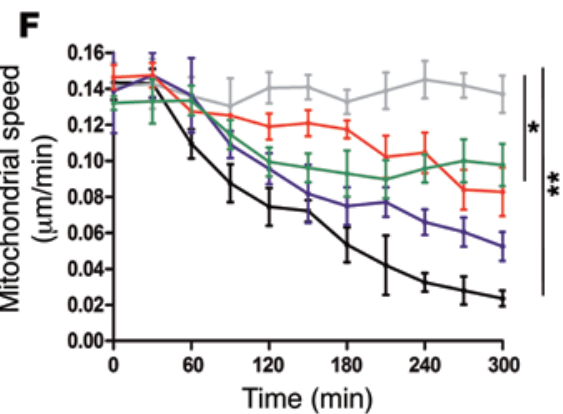

$\mathbf{K}$

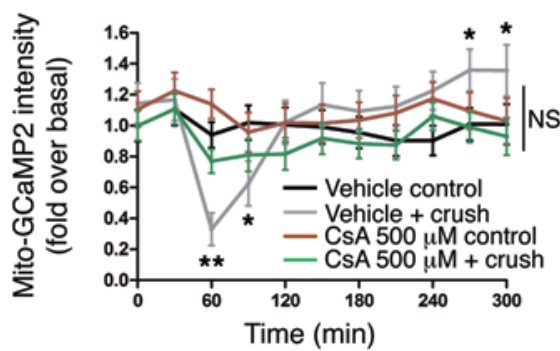

D

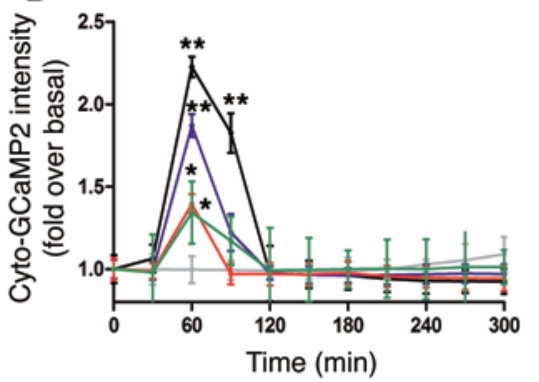

G

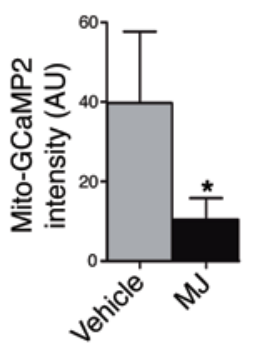

H

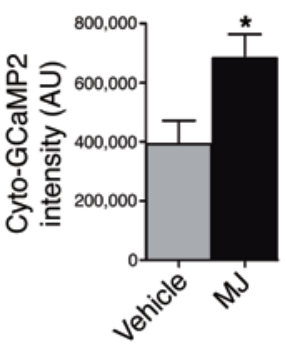

$\mathbf{L}$

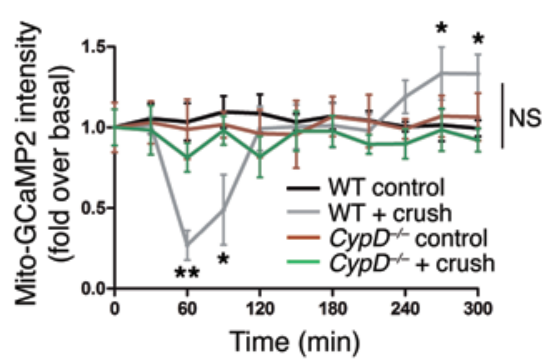

E

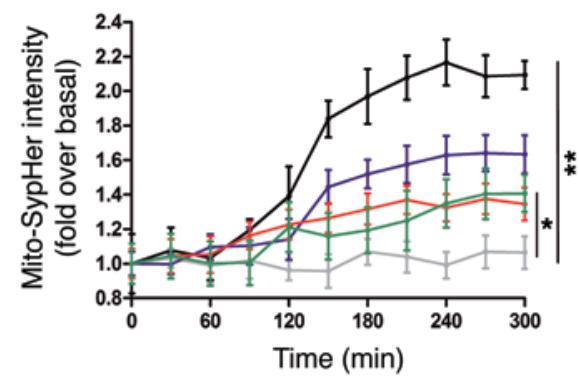

I
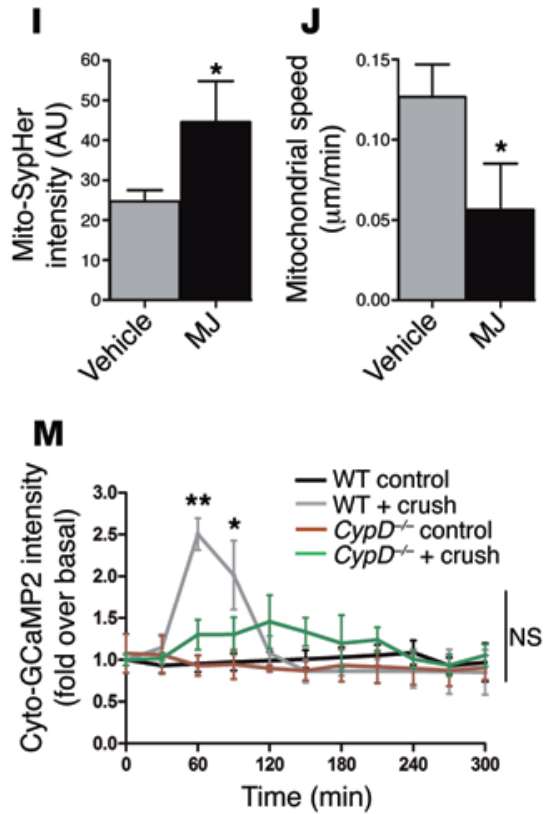

Figure 2. VDAC1 controls mitochondrial calcium release during demyelination. (A) VDAC1 shRNA validation in vitro. Mouse MSC80 cells were transfected with plasmids expressing VDAC1 shRNAs, and VDAC1 protein amounts were quantified by Western blot. GAPDH was used as loading control. $n=4$ independent experiments. (B) AAV-expressing VDAC1 shRNA 2 or 3 or control shRNA together with GFP (green, white arrows) was injected in mouse sciatic nerve (left). Cells expressing VDAC1 shRNAs express less VDAC1 (blue) in their mitochondria (red) than noninfected surrounding cells or cells expressing control shRNA. Scale bar: $10 \mu \mathrm{m}$. Quantification of VDAC1 fluorescence intensity in infected cells (right). (C) Mitochondrial calcium, (D) cytoplasmic calcium, (E) mitochondrial pH, or (F) mitochondrial motility changes in mSCs expressing control shRNA in control conditions (gray) or after crush (black) or expressing VDAC1 shRNAs 2 (blue) or 3 (red) after crush or treatment with TR019622 (TRO) before crush (green). Asterisks mark statistical differences compared with noncrushed nerve. Quantification of (C) mitochondrial calcium, (H) cytoplasmic calcium, (I) mitochondrial pH, or (J) mitochondrial motility in $\mathrm{mSCs} 2$ hours after vehicle or MJ treatment ( $3 \mathrm{mmol}$ ). (K) Mitochondrial calcium changes in mice treated with vehicle or $500 \mu \mathrm{M}$ CsA 30 minutes before crush or in control conditions (noncrushed mice). (L) Mitochondrial calcium and (M) cytoplasmic calcium changes of WT and CypD ${ }^{-/-}$mice after crush or control conditions. Data are expressed as the mean \pm SEM. $n=3-8$ mice for each group. Asterisks mark statistical differences over control conditions (noncrushed nerve). ${ }^{*} P<0.05,{ }^{* *} P<0.01,2$-tailed Student's $t$ test, compared with noncrushed nerves. 
CsA concentration to block mPTP formation in the nerve in vivo was determined using functional competition with auranofin, a selective inductor of $\mathrm{mPTP}$ formation (ref. 30 and Supplemental Figure 3A). We used $500 \mu \mathrm{M}$ CsA to block mPTP before demyelination induction by nerve crush, and we followed the mitochondrial calcium release using mito-GCaMP2-labeled cells. We did not observe any change in mitochondrial calcium under these conditions (Figure $2 \mathrm{~K}$ ), suggesting that MPTP formation is required for the mitochondrial calcium release after nerve injury. To go further and confirm the role of $\mathrm{mPTP}$, we also used a cyclophilin D knockout mouse model (referred to herein as $C y p D^{-1-}$ mice) $(31,32)$. Cyclophilin $\mathrm{D}$ is a protein required for $\mathrm{mPTP}$ formation $(31,32)$. We injected viruses expressing mito-GCaMP2, cyto-GCaMP2, or mito-SypHer into the sciatic nerves of these mice, and then we crushed the nerves in order to follow mitochondrial physiology during demyelination. We found that the loss of the $C y p D$ gene prevented mitochondrial calcium release after crush (Figure 2L) and strongly reduced the concomitant appearance of calcium in the cytoplasm (Figure $2 \mathrm{M}$ ). In addition, the mitochondrial $\mathrm{pH}$ did not change when $\mathrm{mPTP}$ could not form (Supplemental Figure 3B).

Taken together, these data confirm that MPTP formation is required for mitochondrial calcium release during demyelination. The exiting calcium originates from the mitochondrial matrix via MPTP and VDAC1.

The release of mitochondrial calcium via VDAC1 induces demyelination. Since SCs downstream of the nerve crush demyelinate, we hypothesized that the observed mitochondrial calcium release activates demyelination molecular pathways. We injected vehicle or TRO19622 in sciatic nerves before crush or vehicle or MJ in sciatic nerves in absence of crush and determined pathways activation using Western blot. In vehicle-treated samples, an increase in phospho-ERK1/2, phospho-p38, phospho-JNK, and phospho-c-JUN was observed 4 and 12 hours after sciatic nerve injury, and this effect was reduced when nerves were treated with TRO19622 before crush (Figure 3A and Supplemental Figure 4). On the contrary, MJ treatment was sufficient in absence of crush to activate these phosphorylation pathways (Figure 3A and Supplemental Figure 4). Interestingly, total c-JUN and JNK expression increased after nerve crush, and this increase was not blocked by VDAC1 inhibition (Figure 3A). Moreover, when VDAC1 was opened with MJ, expression of c-JUN and JNK did not rise (Figure $3 \mathrm{~A}$ ). Taken together, these data suggest that the activation of ERK1/2, p38, JNK, and c-JUN, but not the increase of total c-JUN and JNK expression, is induced by mitochondrial calcium release through VDAC1.

As mitochondrial calcium release has been involved in cell death and apoptosis (24), we checked mitochondria-related cell death factors in crushed or MJ-treated nerves. Neither phosphoBCL-2 nor cleaved caspase-3 levels increased after nerve crush or MJ treatment (Figure 3A and Supplemental Figure 4), indicating that mitochondrial calcium release does not induce SC death after crush, consistent with previous data that show that demyelination is not a cell death but a dedifferentiation process $(33,34)$.

We also selectively silenced VDAC1 in mSCs and measured nuclear phospho-c-JUN expression by immunohistochemistry after nerve crush. A significant reduction of phospho-c-JUN was observed when VDAC1 was silenced or blocked (Figure 3, B and $\mathrm{C})$, while opening it with MJ was enough to increase nuclear phospho-c-JUN (Figure 3C). However, the total level of c-JUN was not altered during VDAC1 silencing or blocking (Figure 3D), confirming that the increase of total c-JUN is independent of mitochondrial calcium release through VDAC1. Finally, in CypD knockout mice, the activation of the demyelination pathway and nuclear phospho-c-JUN was also blocked (Supplemental Figure 3, C and $\mathrm{D})$, confirming that MPTP formation is also required to induce the molecular demyelination pathway.

Finally, we investigated demyelination by characterizing the cellular morphology of mSCs. In control situations, mSCs displayed a long homogenous noodle-like morphology, with a regular diameter (Figure 3E), while 4 days after nerve crush, demyelinating SCs acquired a heterogeneous morphology, with thin extensions similar to those of non-myelin-forming cells (Figure 3E). After nerve crush, $>90 \%$ of cells expressing control shRNA had a demyelinating phenotype compared with $<10 \%$ of cells without crush (Figure 3, E and F). When VDAC1 was silenced or blocked or when $\mathrm{mPTP}$ could not form (CypD knockout mice), the number of remaining myelinating cells after crush increased significantly (Figure 3, F and G, and Supplemental Figure 3E), whereas a single injection of $\mathrm{MJ}$ in a WT mouse was sufficient to induce demyelination (Figure 3, E and G). This indicates that VDAC1 is responsible for demyelination of mSCs in crushed nerves.

Mitochondrial physiology and VDAC1 activity are altered in SCs of diabetic mice. Diabetic peripheral neuropathy is deeply linked to oxidative stress and mitochondrial dysfunctions (35). However, the causes of diabetic peripheral nerve defects and the role of mitochondria in this neuropathy remain unknown. We hypothesized that defects in SC mitochondrial calcium storage and/or release could be a cause of the demyelinating phenotype associated with this disease. Using the $d b / d b$ diabetic mouse model, we observed that the relative calcium concentration in $\mathrm{mSC}$ mitochondria of diabetic mice was 2.1 times lower than that in control mice (Figure 4A), while the relative cytoplasmic calcium was higher (Figure 4B). In addition, mitochondrial $\mathrm{pH}$ was increased (Figure $4 \mathrm{C}$ ) and mitochondrial motility was decreased (Figure 4D). We then induced demyelination by nerve crushing and observed that mSC mitochondria in diabetic and control mice released calcium at the same time and with a similar kinetic of activation (Figure 4E). However, the level of mitochondrial calcium remained lower for longer time in diabetic SCs. In parallel, calcium appeared in diabetic SC cytoplasm at a similar kinetic as in controls but remained high longer (Figure 4F), suggesting that calcium leaked out of diabetic SC mitochondria, leading to high cytoplasmic calcium and permanent increase in mitochondrial $\mathrm{pH}$ and decrease in organelles motility. This change in calcium homeostasis could therefore underlie reduced $\mathrm{pH}$ response after nerve crush (Figure $4 \mathrm{G}$ ) and limited the drop in mitochondrial motility after nerve injury (Figure 4D). We observed no change in the fusion and fission ratio (Figure $4 \mathrm{H}$ ) or in mitochondrial size (Figure 4, I and J) between diabetic and control mice.

Because the $d b / d b$ mouse model shows morbid obesity associated with diabetes, we wanted to confirm that the mitochondrial changes we saw in this model resulted from diabetes and not obe- 
A

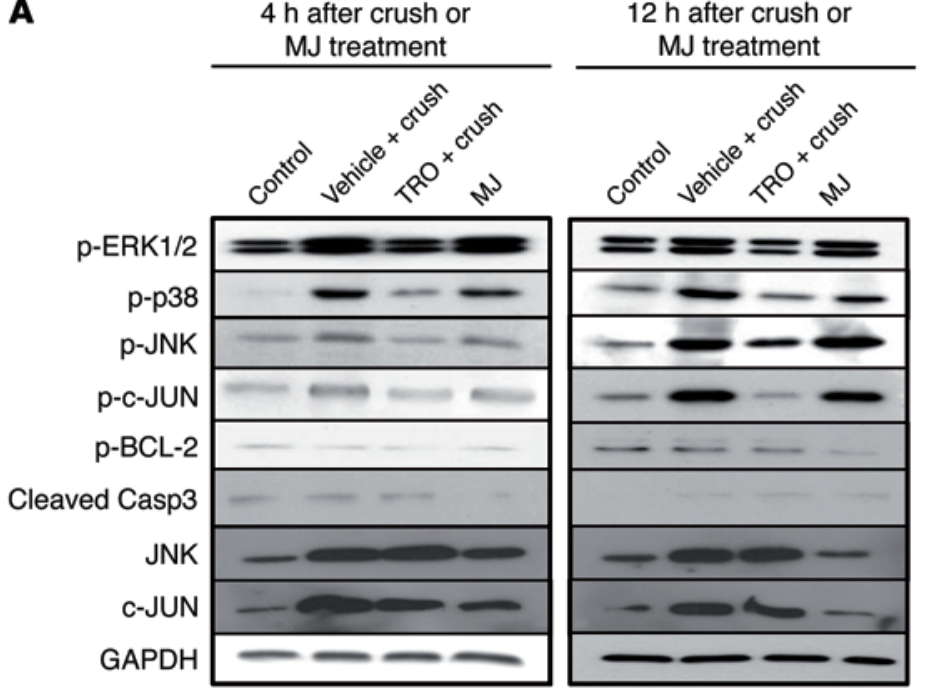

C
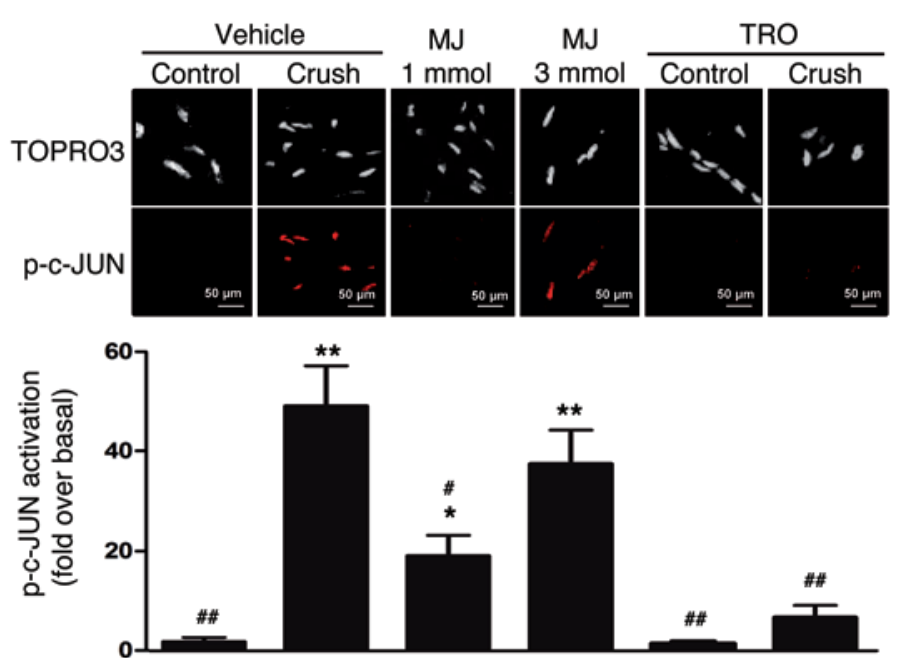

E
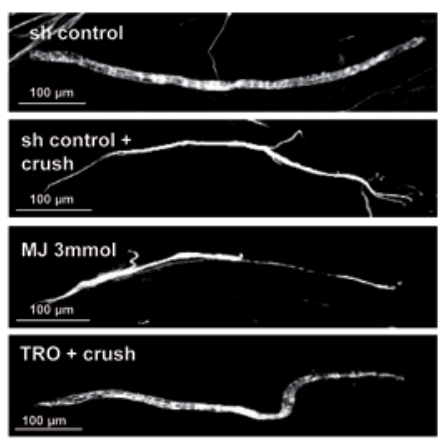

F

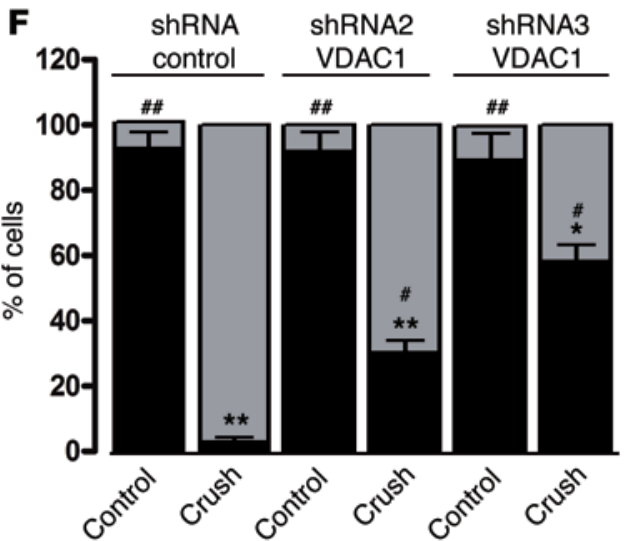

B
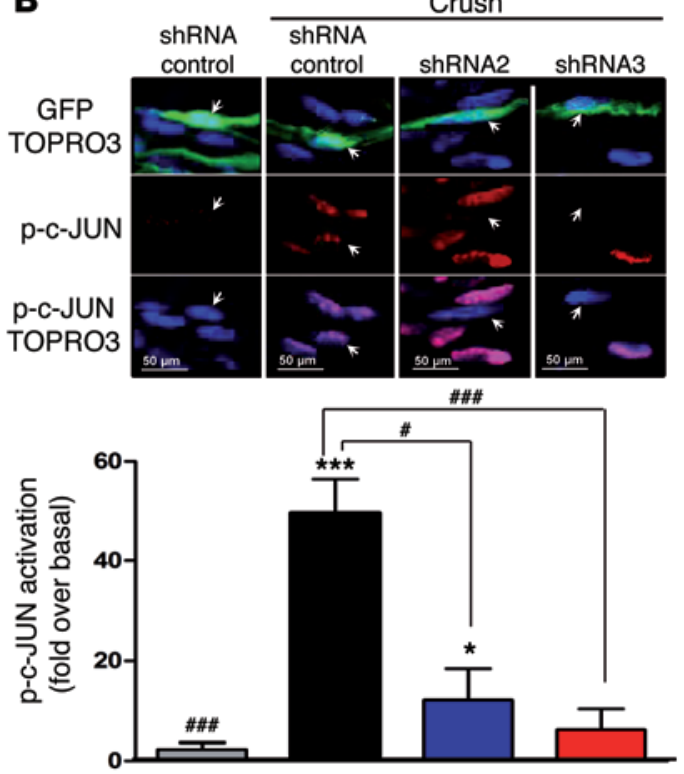

D
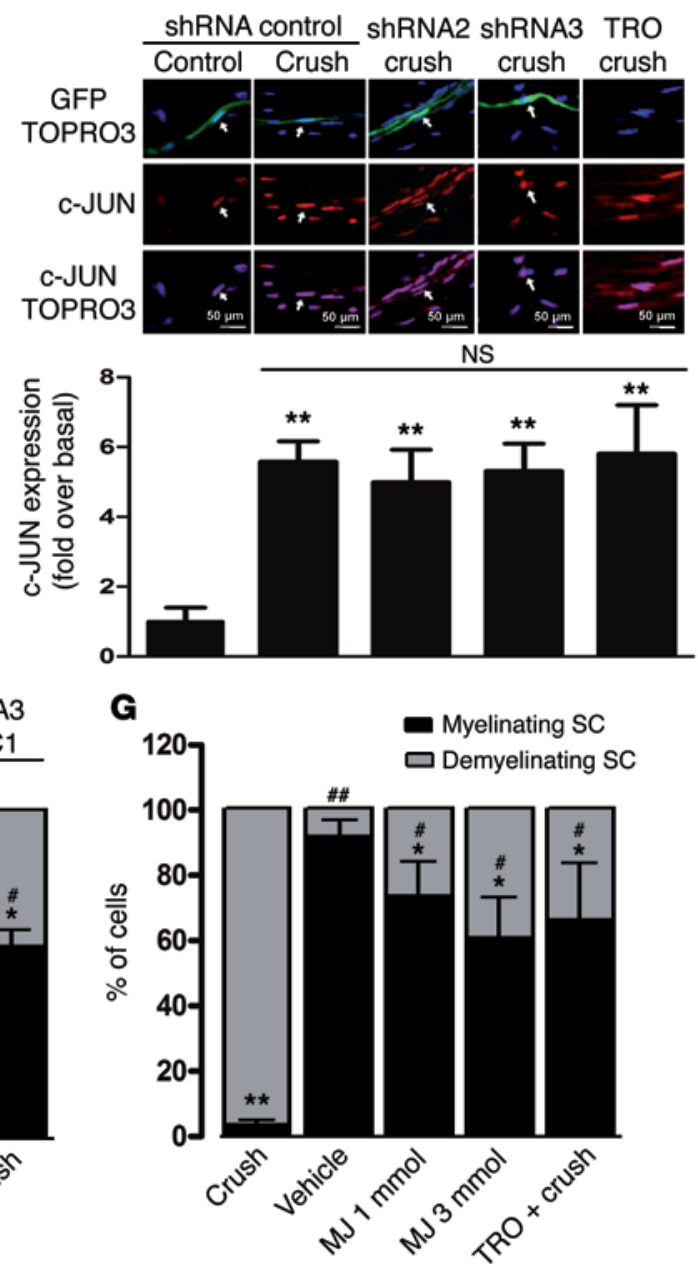
Figure 3. Mitochondrial calcium release through VDAC1 induces SC demyelination. (A) Western blot analysis of phosphorylated ERK1/2, p38, JNK, c-JUN, BCL-2, cleaved caspase-3, total JNK, and c-JUN 4 hours or 12 hours after crush, with or without TR019622 treatment or without crush but with MJ treatment. CAPDH was used as loading control. Blots are from samples run on parallel gels. $n=3-4$ mice for each group. (B and $\mathbf{C}$ ) Immunohistochemistry for nuclear phospho-c-JUN in mSCs of crushed or noncrushed control nerves after (B) VDAC1 silencing or (C) MJ or TR019622 treatment. Mice were treated with TR019622 intraperitoneally for 4 days before crush and via nerve injection 30 minutes before crush or treated with MJ via nerve injection 30 minutes before crush. Nerves were analyzed 12 hours after crush or injection. Representative images are shown. mSCs are stained for nuclei (TOPRO3, blue or white) and phospho-c-JUN (red), and infected cells express GFP (green). Arrows indicate infected mSC nuclei. Scale bar: $50 \mu \mathrm{m}$. Quantification of fluorescence intensity as fold over noncrushed nerve (basal). (D) Immunohistochemistry for total c-JUN in mSCs after VDAC1 silencing (shRNA) or blocking (TR019622) and crush. Arrows indicate VDAC1 shRNA-infected mSC nuclei. Scale bar: $50 \mu \mathrm{m}$ Quantification of total c-JUN represented as fold over basal (noncrushed mice). Noninfected neighbor cells were used as internal controls. (E) Representative images of myelinating and demyelinating SCs expressing GFP after crush or drug treatments. Scale bar: $100 \mu \mathrm{m}$. Quantification of myelinating and demyelinating SC frequency after (F) VDAC1 silencing and (C) MJ and TR019622 treatment. Data are expressed as the mean \pm SEM. $n=3-5$ mice for each group. Asterisks and pound signs mark statistical differences compared with noncrushed and crushed nerves, respectively. ${ }^{*} P<0.05,{ }^{\#} P<0.05$, ${ }^{* *} P<0.01,{ }^{\# \#} P<0.01$, ${ }^{* *} P<0.001$, ${ }^{* \#} P<0.001$, 2-tailed Student's $t$ test.

sity. We repeated the experiments with a NOD mouse model (36). Similar results were obtained with mitochondria in NOD mice (Supplemental Figure 5), showing that the mitochondrial defects we detected were indeed due to diabetes.

Taken together, these data indicate that the physiology of mitochondria is strongly affected in mSCs of diabetic mice. Calcium appears to leak out of mitochondria, resulting in altered levels of cytoplasmic and mitochondrial calcium, increased mitochondrial $\mathrm{pH}$, and reduced mitochondrial movements.

Inhibiting mitochondrial calcium release in SCs prevents demyelinating peripheral neuropathy. We observed that VDAC1 expression in sciatic nerves of $d b / d b$ diabetic mice was not affected at basal conditions (Supplemental Figure 6). To determine whether VDAC1 function is involved in diabetic mSC mitochondrial anomalies, we expressed VDAC1 shRNAs together with fluorescent probes in diabetic mSCs. After 3 weeks of VDAC1 silencing, we observed a significant increase in relative mitochondrial calcium concentration and a proportional decrease in cytoplasmic calcium (Figure 5, A and B). Moreover, we observed similar changes when diabetic mice were treated for 4 days with TRO19622 (Figure 5, A and B), showing that VDAC1 is responsible for the calcium leak to the cytoplasm. Finally, reducing mitochondrial calcium leak by VDAC1 silencing or TRO19622 inhibition significantly reduced mitochondrial pH (Figure 5C) and increased mitochondrial motility (Figure 5D). Therefore, silencing VDAC1 expression or inhibiting VDAC1 function can prevent the consequences of calcium leakage in diabetic mSCs.

Diabetic peripheral neuropathy in humans is characterized by a chronic demyelination (9). In diabetic mouse models, peripheral nerve defects (thinner myelin, reduced NCV, motor impairment) and activation of demyelination signaling can be detected at early adulthood $(37,38)$. However, demyelination is not significant before
1 to 1.5 years and therefore overlaps with aging-induced peripheral nerve defects $(39,40)$. We therefore chose not to focus on demyelination itself but on early peripheral nerve defects in diabetic mice.

We observed high expression levels of nuclear phosphoc-JUN in diabetic mSCs that were strongly reduced by VDAC1 silencing or blocking (Figure 5, E and F). We then treated diabetic and control mice daily with vehicle or TRO19622 for 1 month and evaluated myelin using electron microscopy 1 day and 2 months after drug treatment (Figure 5G and Supplemental Figure 7). We observed a significant decrease in g-ratio (axon diameter/total fiber diameter) of diabetic mice treated with TRO19622 compared with that of those treated with vehicle (Figure 5, H and I), while no difference was found in axon number and diameter (Figure 5I), indicating that the inhibition of mitochondrial calcium release partially normalized myelin thickness in diabetic mice.

NCV is reduced in diabetic mice $(37,38,41)$. AfterVDAC1-blocking treatment, the NCV significantly increased, reaching control values (Figure 6, A and B) without change in compound muscle action potential (CMAP) amplitude (Figure 6, A and B).

Finally, we evaluated neuromuscular performances of diabetic mice. A significant increase in rotarod latency and grip strength was observed 20 days after TRO19622 treatment (Figure 6C), but no change was found in CatWalk test parameters (Supplemental Figure 8). These recoveries were not due to change in weight (Supplemental Figure 9).

We then sought to test whether the inhibition of mitochondrial calcium release could also prevent demyelination in demyelinating forms of inherited neuropathies. We chose the CMT1A rat $(42,43)$ as a representative animal model of CMT1A, the most prevalent demyelinating form of CMT in humans (44). CMT1A rats were treated daily with TRO19622 for 15 or 30 days and analyzed 1 day or 2 months after treatment. We observed an activation of phospho-ERK1/2, phospho-p38, phospho-JNK, and phosphoc-JUN in basal conditions, and, after 15 and 30 days of TRO19622 treatment, we observed a significant downregulation of these pathways (Figure 7A and Supplemental Figure 10). However, these effects disappeared 2 months after stopping the treatment.

TRO19622 treatment strongly reduced the amount of phospho-c-JUN in CMT1A rat mSC nuclei (Figure 7B), further suggesting that blocking VDAC1 function prevents activation of demyelination pathways. Indeed, the number of demyelinated fibers (Figure 7C) and the g-ratio significantly decreased (Figure 7, D and E, and Supplemental Figure 11) and the number and diameter of nerve axons increased (Figure 7E) in TRO19622-treated rats. Finally, we analyzed nerve conduction in rats treated with TRO19622 for 1 month, and we observed a significant increase of CMAP amplitude and NCV (Figure 7, F and G). Concomitantly, the functional tests in treated rats revealed an increase in accelerating rotarod latency (Figure $7 \mathrm{H}$ ) and grip strength (Figure 7I and Supplemental Figure 12), a decrease in the amount of time animals stand on each of their paws (Figure 7J) and and distance between hind paws (Figure $7 \mathrm{~K}$ ), and an increase in paw print area (Figure $7 \mathrm{~L}$ and Supplemental Figure 13). All of these changes indicated a functional recovery of the motor performances of the TRO19622treated CMT1A animals. This recovery was not due to change in the body mass (Supplemental Figure 12). Nevertheless, the effects of TRO19622 on demyelination, g-ratio, and behavioral motor 

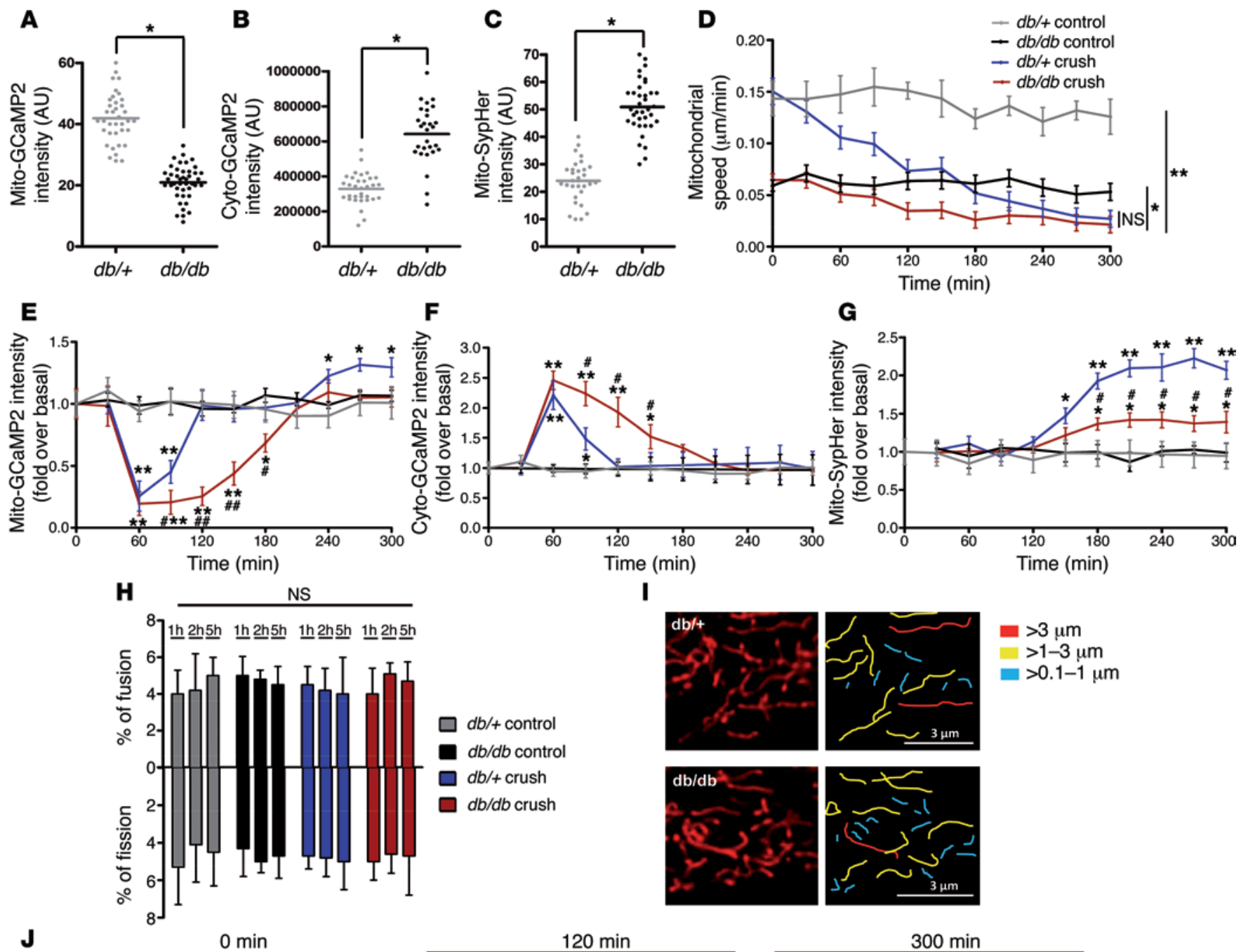

I
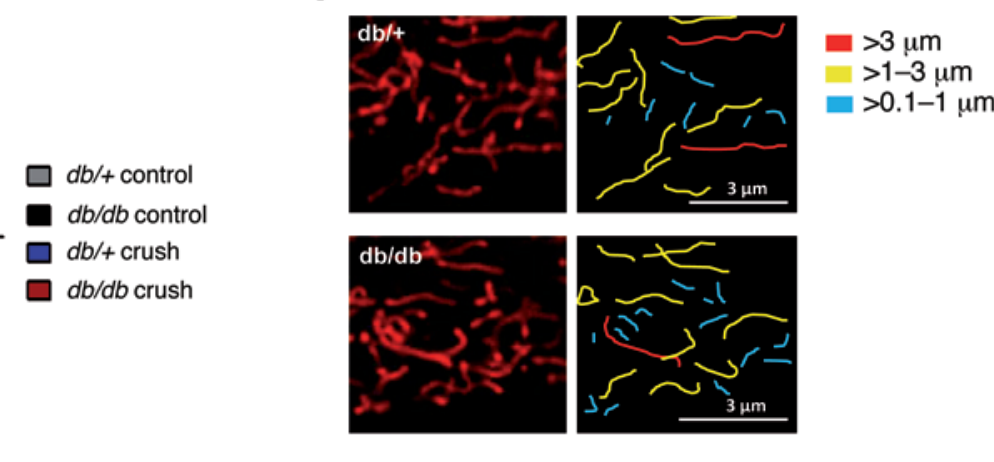

$300 \mathrm{~min}$

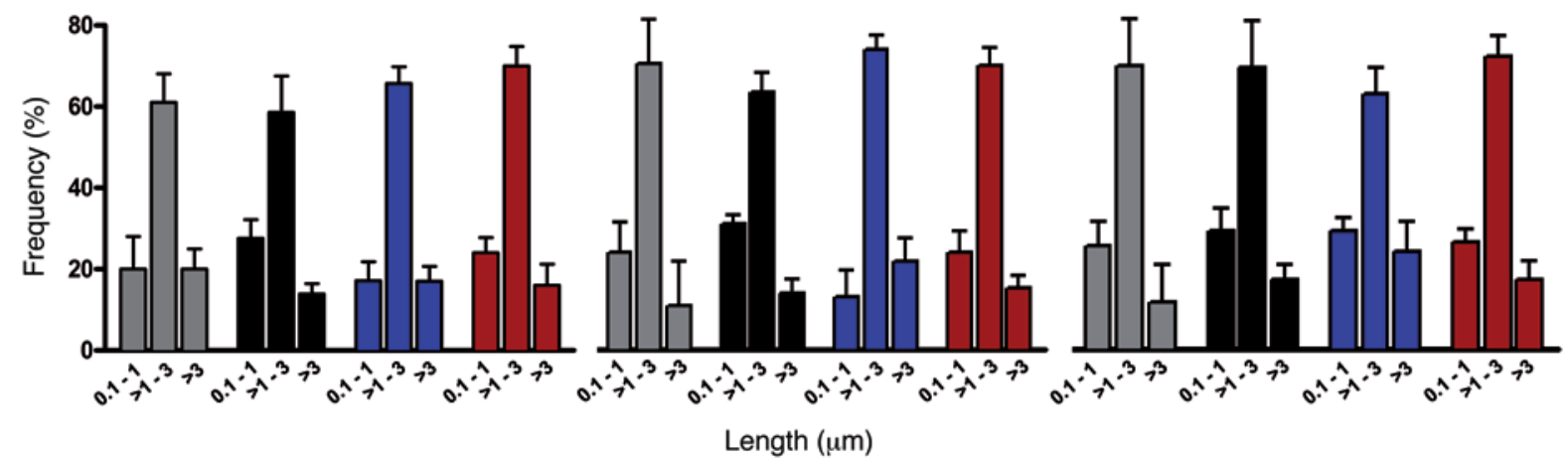

Figure 4. Mitochondrial physiology is altered in SCs of $\boldsymbol{d b} / \mathbf{d} \boldsymbol{b}$ diabetic mice. (A) Mitochondrial calcium, (B) cytoplasmic calcium concentration, and (C) mitochondrial pH in mSCs of control $(d b /+)$ and diabetic $(d b / d b)$ mice in basal conditions. (D) Mitochondrial motility, (E) mitochondrial calcium, (F) cytoplasmic calcium concentration, and (G) mitochondrial pH changes after crush or in control noncrushed conditions in mSCs of control and diabetic mice. (H) Ratio of mitochondrial fusion and fission events in mSCs of control and diabetic mice in crushed or control noncrushed conditions. (I) Representative images of mSC mitochondria (left panels) and mitochondrial length quantification (right panels) of control and diabetic mice after 300 minutes of time-lapse imaging acquisition. Scale bar: $3 \mu \mathrm{m}$. (J) mSC mitochondrial length frequency of control and diabetic mice in crushed or control conditions at different time points. No significant difference was found between control $(d b /+)$ and diabetic $(d b / d b)$ mice. Data are expressed as the mean \pm SEM. $n=3-6$ mice. Asterisks and pound signs mark statistical differences compared with noncrushed control mice and crushed control mice, respectively. ${ }^{*} P<0.05,{ }^{\#} P<0.05,{ }^{* *} P<0.01,{ }^{\# \# P}<0.01$, 2-tailed Student's $t$ test. 
function disappeared 2 months after stopping the treatment (Supplemental Figure 14). Taken together, these data show that inhibition of VDAC1 function prevents the activation of demyelination pathways, improves myelination, preserves axons, and increases nerve conduction and neuromuscular performances in CMT1A rats. Therefore, inhibiting mitochondrial calcium release through VDAC1 represents a target for the treatment of CMT1A.

\section{Discussion}

While demyelinating peripheral nerve diseases include a large spectrum of disabling acquired and inherited diseases, the mechanisms of demyelination remain elusive. Demyelination is a cellular program that is triggered in mSCs upon encounter with specific signals, such as axonal injury in a trauma. These triggering signals are transduced in the cell, leading to the recruitment of phosphorylated c-JUN in the nucleus $(45,46)$. This gene participates in the demyelination program in the nucleus and mediates a protective effect against loss of sensory axons (47). Here, we describe a mitochondrial molecular mechanism leading to SC demyelination in peripheral neuropathies. We show that the release of calcium by mitochondria through VDAC1 is the earliest step recorded after nerve injury and that this step is necessary and sufficient for triggering the SC demyelination program (Figure 8).

SC demyelination is initiated by crushed or severed axons after nerve injury, and the initial signal involved is unknown. As VDAC1 and other calcium channels are likely to be expressed in the plasma membrane of mSCs (48), it is possible that the activation of these channels by axonal cues also contributes to the cytoplasmic calcium increase in mSCs following nerve crush. While we did not detect such a cytoplasmic calcium signal after nerve crush and prior to the mitochondrial calcium release, it is worth noting that our measurements may have been taken at times that were too close together to allow the detection of a short and moderate calcium wave. This question of the nature of the original signal that initiates demyelination is therefore still pending.

How the burst of mitochondrial calcium activates the ERK1/2, p38, and JNK pathways through VDAC1 channels is not clear. However, cytoplasmic calcium stimulates ERK1/2 (49) and JNK (50) activity. Several studies also reported an association of ERK1/2, p38, and JNK and their direct activators, such as Raf, Sab, and MKK4, with mitochondria (51). So it is likely that mitochondrial calcium release in the cytoplasm directly activates MAPK demyelination pathways, thus propagating the demyelination signal all over the cell.

VDAC1 has numerous binding partners that control its permeability, in particular, hexokinase (HK). HK binding to VDAC1 reduces the permeability of the pore, notably, to calcium (25). In SCs, MJ, a compound that uncouples HK from VDAC1 (27), induced mitochondrial calcium release and demyelination, showing that $\mathrm{HK}$ is essential to control demyelination. Intriguingly, mutations in the HK gene are responsible for demyelinating CMT4G disease (52), suggesting that an increased permeability of VDAC1 to calcium is the cause of the disease.

Peripheral neuropathy is a major complication of diabetes, and, while the pathomechanism of the disease is unknown, the role of mitochondria in diabetic neuropathy remains poorly studied (17). We investigated whether mitochondrial physiology was altered in mSCs before and after nerve injury in obese diabetic $d b / d b$ mice but also in NOD mice $(39,53)$. We found that, in both models, VDAC1 activity was altered, allowing for calcium leak in diabetic mouse mSCs. This higher amount of mitochondrial calcium signaling constitutively stimulated MAPK demyelination pathways and increased nuclear phospho-c-JUN, priming mSCs for demyelination.

Peripheral nerve demyelination can be initiated by various mechanisms, including nerve injury, metabolic alterations, and gene mutations. Such heterogeneity has hindered the search for a common treatment for these peripheral neuropathies. Our results show that blocking mitochondrial calcium release prevented demyelination in a classical nerve crush injury but also in a diabetic model, suggesting that the mitochondrial step toward demyelination is common to different pathologies. To confirm this, we used another model of demyelination, the CMT1A rat (42). We showed that mitochondrial calcium release through VDAC1 is also involved in CMT1A rat demyelination. These data indicate that the mitochondrial step is likely to be involved in other demyelinating peripheral neuropathies.

We noted that the effects of VDAC1 inhibition on MAPK activation, nerve structure, and neuromuscular performance did not last 2 months after stopping the treatment in both diabetic mice and CMT1A rats (Supplemental Figures 9 and 14). This suggests that mitochondrial calcium release is constitutive in these chronic diseases and transiently inhibiting VDAC1 activity cannot provide a definitive relief. In addition, TRO19622 was originally described for its effects on neurons (26), and its use in recent trials in phase II/III for motor neuron disease/amyotrophic lateral sclerosis has been reported (54). So we cannot exclude an effect of TRO19622 on axonal mitochondria that could indirectly support SC myelination. In contrast, an indirect effect of the drug on neurons via myelin mitochondria cannot be excluded to our knowledge. In this regard, it has been reported that SCs expressing a mutant SOD1 may provide support for motor neurons of the ALS mouse model (55). In any case, as inhibition of VDAC1 through TRO19622 has been shown to be safe in humans (54), new drug design or existing drug improvement could be an attractive possibility to generate a treatment with long-lasting benefit for patients with CMT. Taken together, our data bring to light a molecular mechanism that launches demyelination in SCs. They also show that blocking this mechanism may represent a new opportunity for treatment for patients suffering from hereditary and acquired demyelinating peripheral neuropathies.

\section{Methods}

Additional details are available in the Supplemental Methods.

\section{Cloning}

Plasmid dsRed2-mito7 (Clontech, 55838) was cut using NheI and NotI enzymes and then treated with a DNA polymerase I large Klenow fragment (New England Biolabs, M0210S) to isolate mito-dsRed2 cDNA. After purification, it was cloned into pAdtrack-CMV (Quantum Biotechnologies Inc.) or pAAV-MCS (Cell Biolabs Inc.) plasmids under the control of a CMV or a CAG promoter, respectively. pcDNA3.1 mitoGCaMP2 (provided by X. Wang, Peking University, Beijing, China) was cut with HindIII and EcoRV to be cloned into a pShuttle-CMV (Quan- 
A
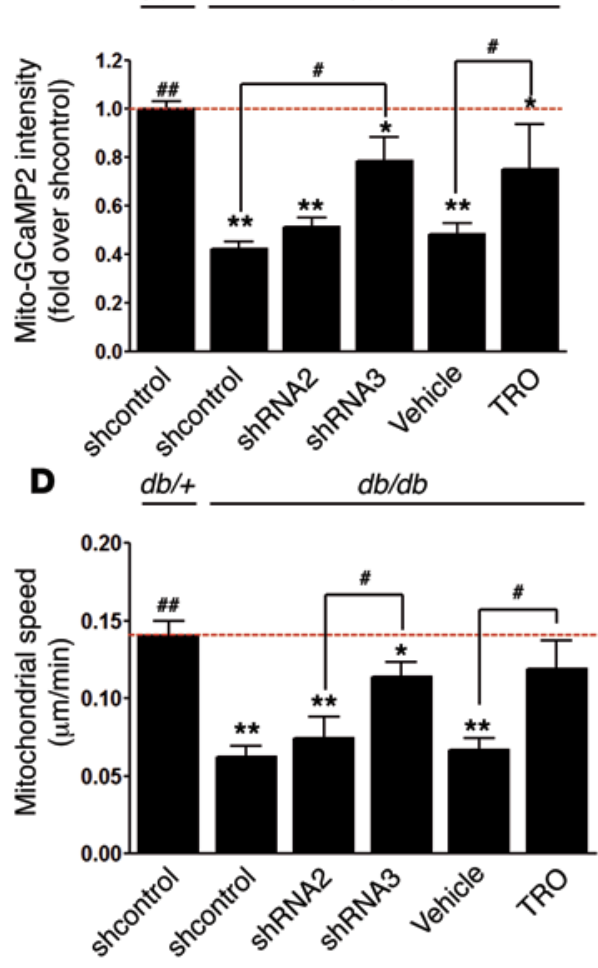

F
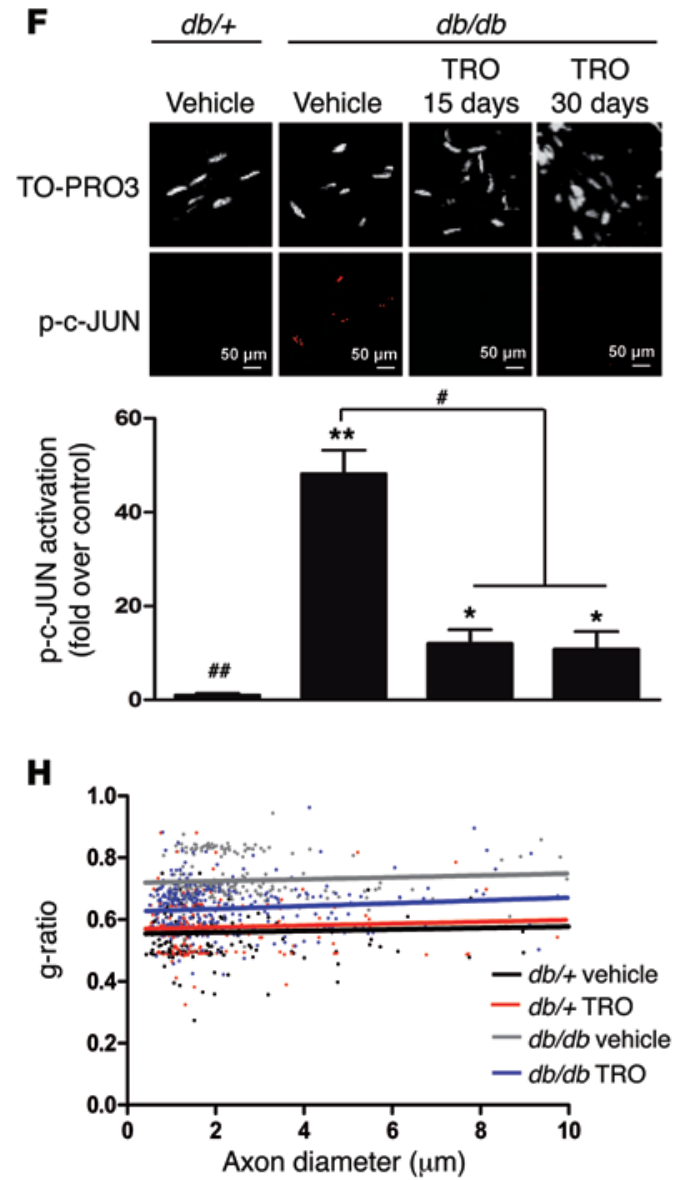

B
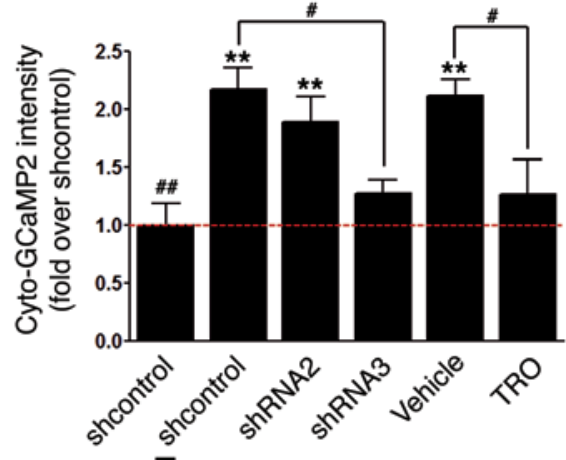

$E$
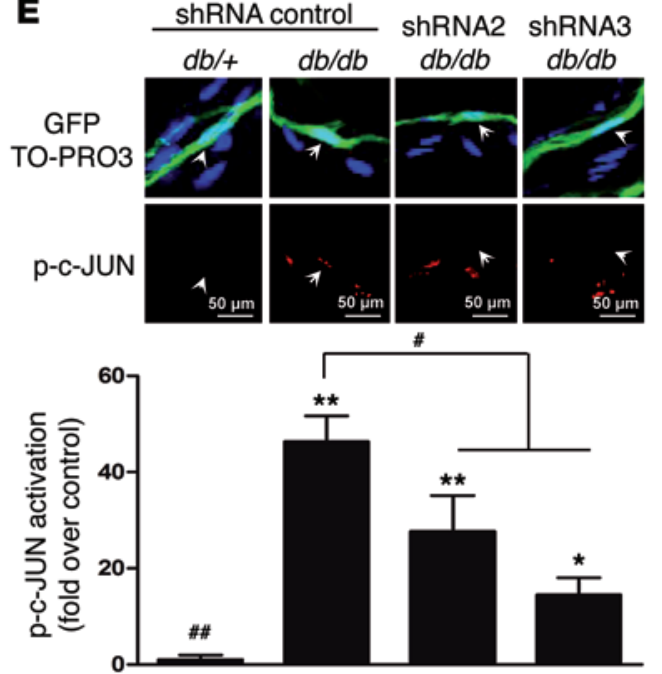

G

Vehicle

TRO
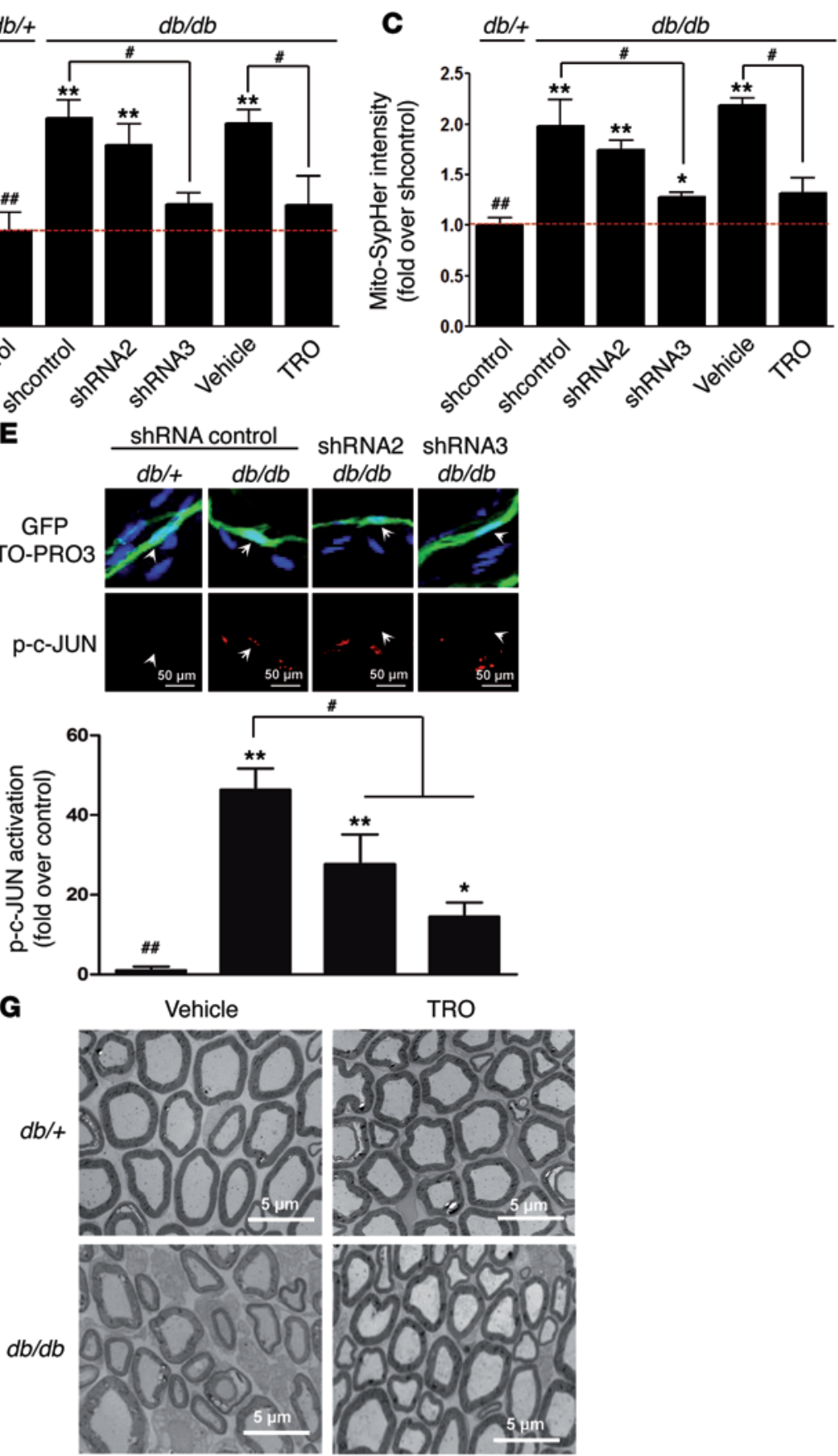

I
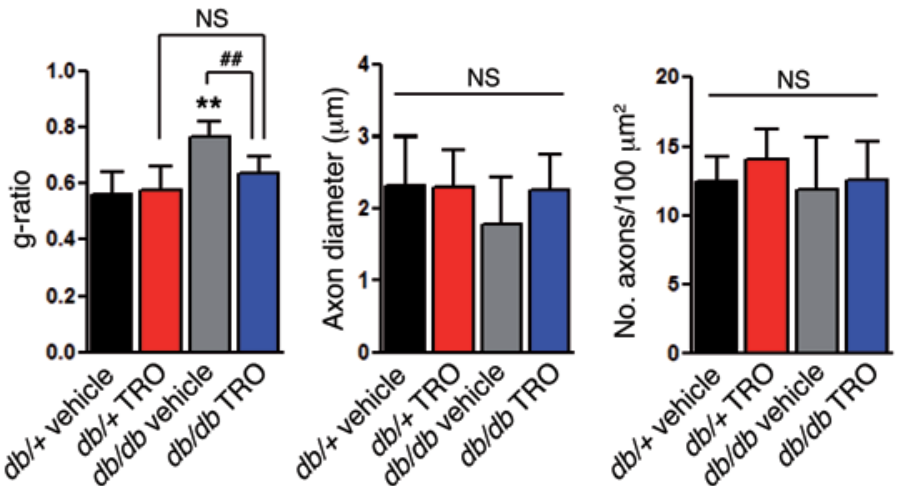
Figure 5. VDAC1 silencing and inhibition prevent SC mitochondrial anomalies and improve the phenotype of diabetic mice. (A) Mitochondrial calcium, (B) cytoplasmic calcium, (C) mitochondrial pH, and (D) motility in $\mathrm{mSC}$ of diabetic mice $(d b / d b)$ in basal conditions are partially corrected during VDAC1 silencing or blocking. Immunohistochemistry for phosphoc-JUN in mSCs of control and diabetic mice after (E) VDAC1 silencing or (F) TR019622 treatment. Arrows indicate infected mSC nuclei. Scale bar: $50 \mu \mathrm{m}$. Quantification of nuclear phospho-c-JUN represented as fold over control mice $(d b /+)$. Noninfected neighbor cells were used as internal controls. (C) Representative transmission electron micrograph images of sciatic nerve cross sections of control $(d b /+)$ and diabetic $(d b / d b)$ mice after vehicle or TR019622 treatment. Scale bar: $5 \mu \mathrm{m}$. (H) Scatterplot showing the g-ratio plotted against the axon diameter of control and diabetic mice treated with vehicle or TR016922 for 30 days. (I) Average myelin g-ratio, axonal diameter, and number of myelinated axons in sciatic nerves of control and diabetic mice described in $\mathbf{H}$. A minimum of 200 fibers was measured per animal. ${ }^{*} P<0.05,{ }^{\#} P<0.05,{ }^{* *} P<0.01,{ }^{\# \# P}<0.01,1$-way ANOVA followed by a Dunnett's multiple comparison post-hoc test.

tum Biotechnologies Inc.) or with BamHI and EcoRV to be cloned into a pAAV-MCS vector under the control of a CMV or a CAG promoter, respectively. Then, the GCaMP2 probe cDNA (without mito sequence) was cut using HindIII and EcoRV and then cloned into a pAAV-MCS and pShuttle-CMV vector. The probe mito-SypHer (provided by J.C. Jonas, Université Catholique de Louvain, Louvain-la-Neuve, Belgium) was cut using NheI and NotI enzymes and treated with DNA polymerase I large Klenow fragment. After purification, it was cloned into pAAV-MCS under the control of a CAG promoter. The VDAC1 shRNA sequence 2, GTTGGCTATAAGACGGATGAACT (Sigma-Aldrich, TRCNO000012391); the VDAC1 shRNA sequence 3, ACCAGGTATCAAACTGACGTTCT (Sigma-Aldrich, TRCNO000012392); or the shRNA control (dsRed2) AGTTCCAGTACGGCTCCAA or (GFP) CAAGCTGACCCTGAAGTTC was first cloned into a pSicoR vector
(Addgene, 11579) under the control of a U6 promoter using HpaI and BstEII enzymes. Then, the U6-VDAC1 shRNA sequences were cut using ApaI and BstEI and then cloned into the pAAV-CMV-GFP vector (Cell Biolabs Inc.), the pAAV-mito-GCaMP2, the pAAV-GCaMP2, the pAAV-mito-dsRed2, or the previously described pAAV-mito-SypHer vector. All clones were validated by sequencing.

\section{Animal housing}

Immunodeficient CB17/SCID mice (Janvier Labs), $d b / d b$ diabetic mice [homozygous BKS(D)-Lepr ${ }^{\mathrm{db}+/+} /$ JOrlRj, Janvier Labs], their controls [heterozygous BKS(D)-Lepr ${ }^{\mathrm{db}+/} / \mathrm{JOrlRj}$, Janvier Labs], NOD mice (NOD/ShiLtJ, 001976, The Jackson Laboratory), CypD $D^{-/-}$mice (B6,129-Ppif ${ }^{\mathrm{m} 1 \mathrm{Imol}} / \mathrm{J}$, 009071, The Jackson Laboratory), and their controls were kept in the A2 animal house facility, whereas transgenic PMP22 CMT1A rats (provided by M. Sereda, Max Planck Institute of Experimental Medicine, Göttingen, Germany) were housed in the A1 animal house facility of the Institute for Neurosciences of Montpellier. Mice were housed in ventilated and clear plastic boxes and subjected to standard light cycles (12 hours in 90-lux light, 12 hours in the dark). Experiments were realized at 8 weeks of age for CB17/SCID and $C y p D^{-/-}$mice, at 20 weeks of age for $d b / d b$ diabetic and NOD mice, and at 12 weeks of age for CMT1A rats.

\section{Genotype analysis}

Diabetic mice that were homozygous for a leptin mutant [BKS(D)Lepr $^{\mathrm{db}+/+} /$ JOrlRj] and their heterozygous controls [BKS(D)-Lepr ${ }^{\mathrm{db}+/-}$ / JOrlRj] in a C57BL/KS-J background were purchased from Janvier Labs. Homozygous NOD (NOD/ShiLtJ) mice and $C y p D^{-/-}\left(\mathrm{B} 6,129-\mathrm{Ppif}^{\mathrm{m} 1 \mathrm{Jmol}} / \mathrm{J}\right)$ mice were purchased from The Jackson Laboratory. The generation of Pmp22 transgenic CMT1A rats has been previously described in Sereda et al. (42). Routine genotyping for the presence of the Pmp22 transgene was
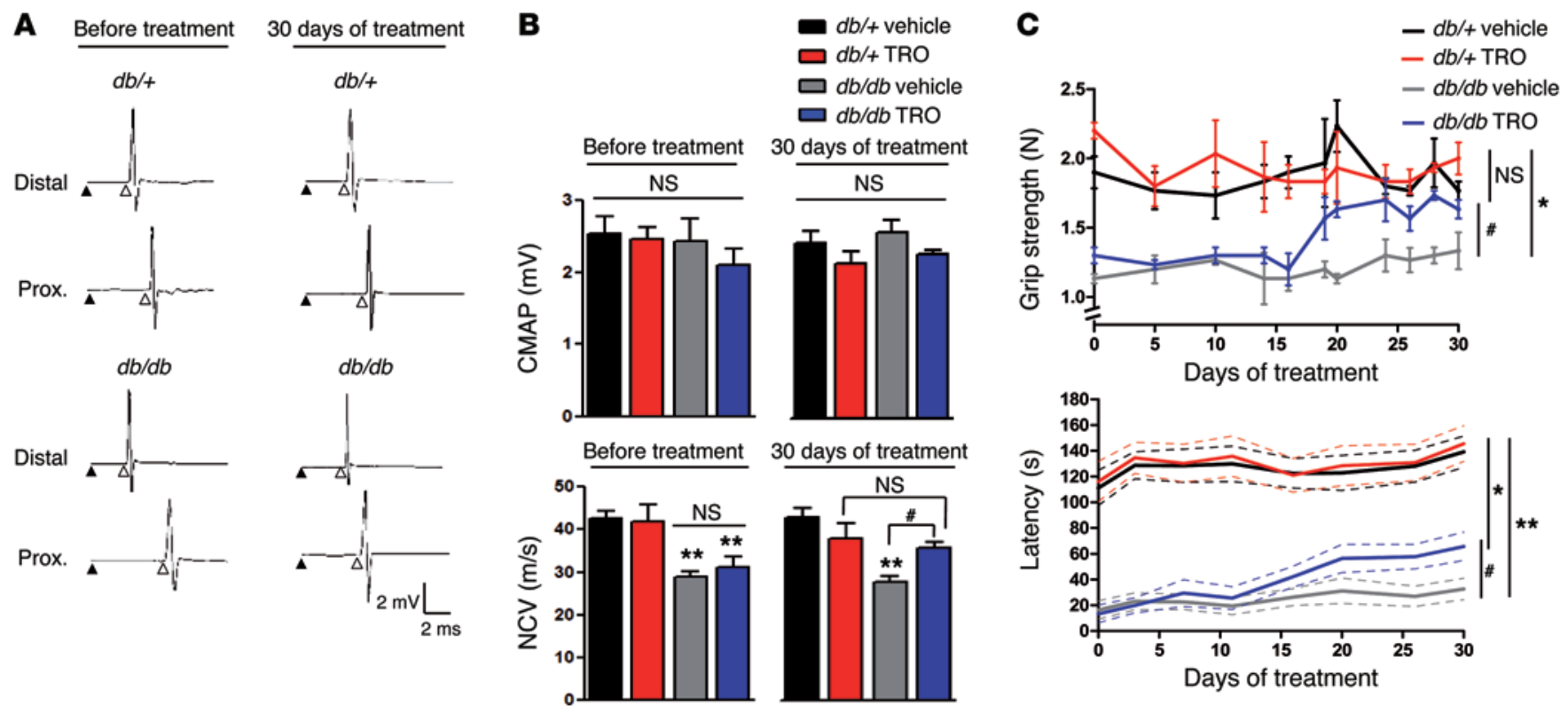

Figure 6. VDAC1 inhibition improves the phenotype of diabetic mice. (A) Representative electrophysiological traces of sciatic nerve recording after distal and proximal stimulation of control and diabetic mice before and after TR019622 treatment. The stimulus artifact (black arrowhead) and the onset of CMAP (white arrowhead) are shown. (B) Quantification of the CMAP and NCV from A. (C) Grip strength and rotarod latency of control and diabetic mice treated with vehicle or TR019622. Data are expressed as the mean \pm SEM. $n=12$ mice for each group. Asterisks and pound signs mark statistical differences compared with control ( $d b /+)$ mice and vehicle-treated diabetic mice, respectively. ${ }^{*} P<0.05,{ }^{*} P<0.05,{ }^{*} P<0.01,1$-way ANOVA followed by a Dunnett's multiple comparison post-hoc test. 


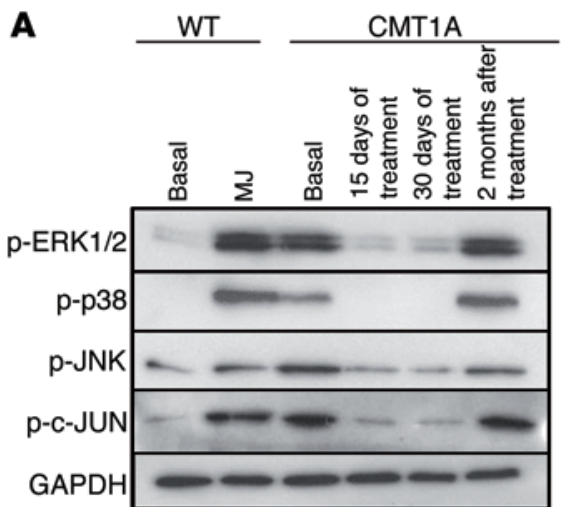

D

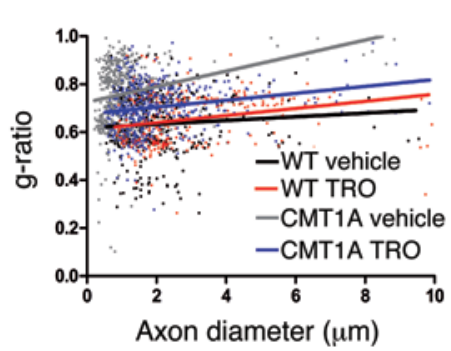

E
B
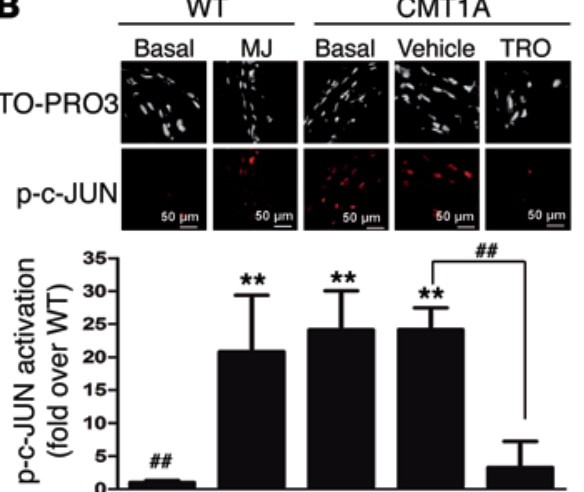
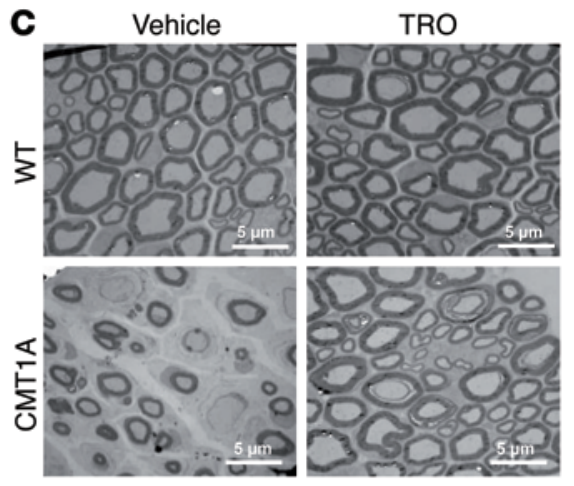

F Before treatment 30 days of treatment
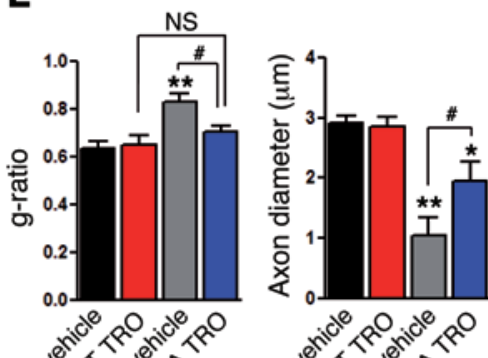

$34 \sin ^{2}$

0,0

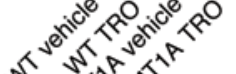

and
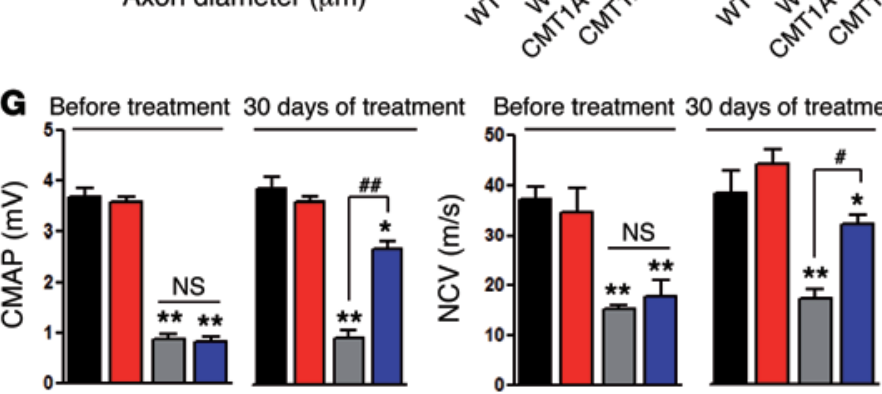

WT vehicle WT TRO $\square$ CMT1A vehicle $\square$ CMT1A TRO

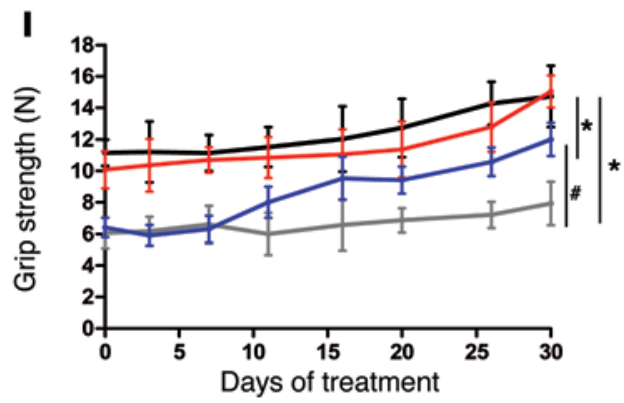

K Before treatment 15 days of treatment 30 days of treatment
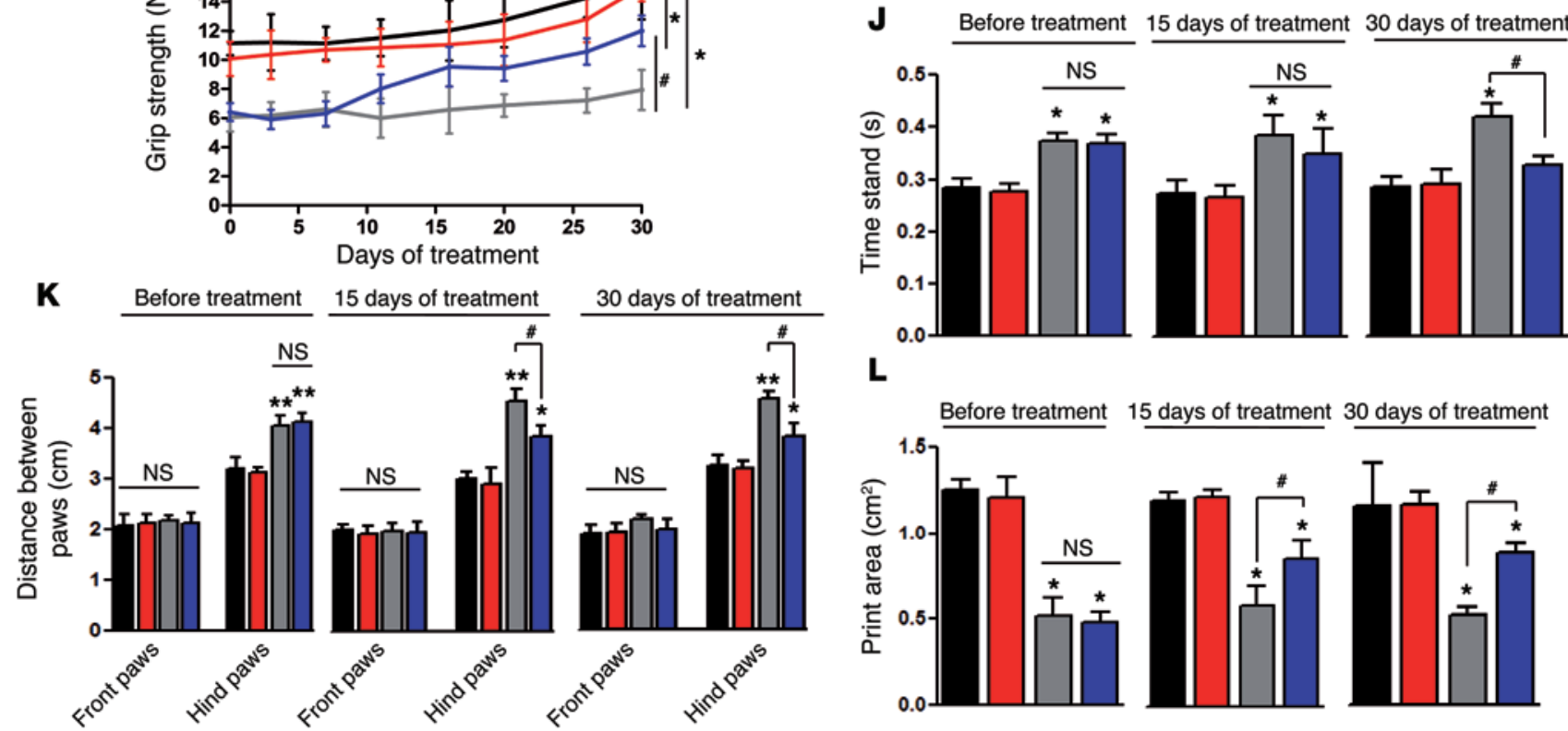

$\mathbf{L}$

Before treatment 15 days of treatment 30 days of treatment
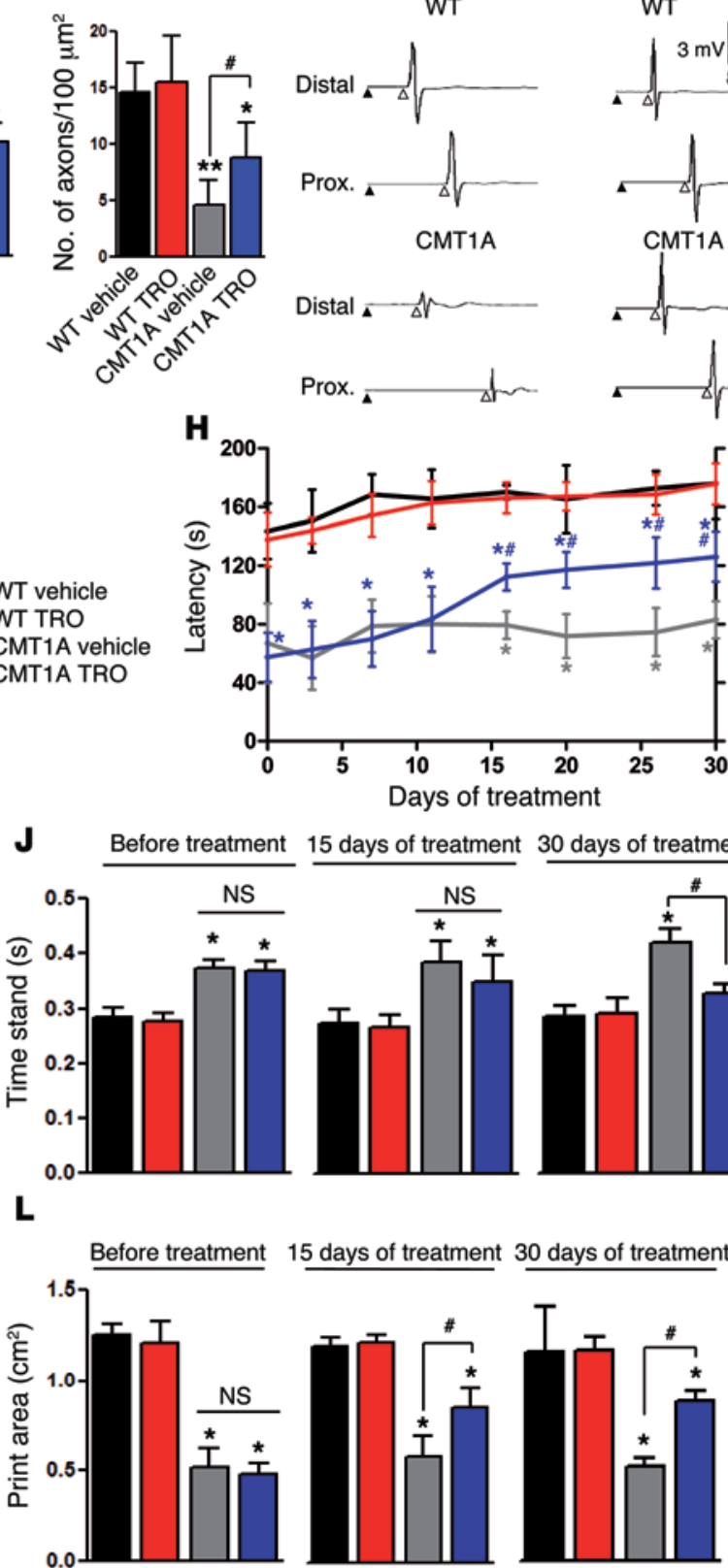

20
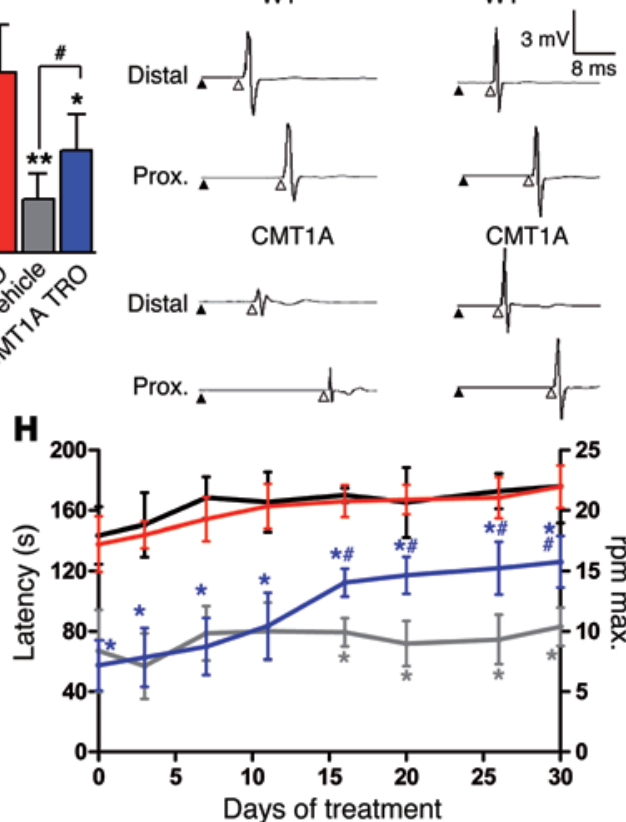
흥 $\frac{\sqrt{3}}{3}$ 
Figure 7. Blocking VDAC1 activity prevents demyelination and improves neuromuscular performance in the CMT1A rat model. (A) Sciatic nerve Western blot analysis of WT and CMT1A rats in basal conditions, WT rats treated with MJ, and CMT1A rats treated with TR019622 for 15 or 30 days or 2 months after ending treatment. GAPDH was used as loading control. $n=3$ rats for each treatment. (B) Immunohistochemistry for phosphoc-JUN in mSCs of WT and CMT1A rats in basal conditions, WT rats treated with MJ, and CMT1A rats treated with vehicle or TR019622 for 30 days. Scale bar: $50 \mu \mathrm{m}$. Quantification of phospho-c-JUN intensity. $n=3-6$ rats for each treatment. (C) Representative transmission electron micrograph images of sciatic nerve cross sections of WT and CMT1A rats after vehicle or TR019622 treatment. Scale bar: $5 \mu \mathrm{m}$. (D) Scatterplot showing the g-ratio plotted against the axon diameter of sciatic nerve fibers from WT and CMT1A rats after vehicle or TR016922 treatment. $n=3$ rats for each group. (E) Average myelin g-ratio, axonal diameter, and myelinated axon number in WT and CMT1A rats described in D. (F) Representative electrophysiological traces of sciatic nerve recording after stimulation of WT and CMT1A rats before and after TR019622 treatment. Stimulus artifact (black arrowhead) and CMAP onset (white arrowhead) are shown. (G) Quantification of CMAP and NCV from F. (H) Accelerating rotarod latency, (I) grip strength, (J) time animals stand on each of their paws (time stand), (K) distance between paws, and (L) paw area of WT and CMT1A rats during vehicle or TR019622 treatments. $n=6-10$ rats for each group. Data are expressed as mean $\pm \mathrm{SEM}$. Asterisks and pound signs mark statistical differences compared with control and CMT1A rats treated with vehicle, respectively. ${ }^{*} P<0.05,{ }^{\#} P<0.05,{ }^{* *} P<0.01,{ }^{\# \# P}<0.01,1$-way ANOVA followed by a Dunnett's multiple comparison post-hoc test.

performed by PCR, using genomic DNA from tail biopsies and the transgene-specific primers 5'-GACAAACCCCAGACAGTTG-3' and 5'-CCAGAAAGCCAGGGAACT-3' under standard conditions.

\section{Viral particle production}

Adenoviral particle production was described in He et al. (56). Briefly, for adenovirus production, pAdtrack vector containing the constructs was recombined with pAdeasy1 vector in the Adeasy1 BJ5183 bacteria strain (Stratagene). The isolated adenoviral DNA was cut with PacI enzyme and transfected in HEK 293 cells using Lipofectamine 2000. The initial production of adenovirus was followed by 3 rounds of amplification. Finally, freeze-thaw cycles were used to harvest the viral particles from cells, and these particles were then purified using cesium chloride gradients. To produce high-titer adeno-associated virus (AAV10), three 15-cm dishes of 70\% to 80\% confluent HEK293T cells were transfected with $71 \mu \mathrm{g}$ pAAV expression vector, $20 \mu \mathrm{g}$ pAAV10 capsid, and $40 \mu \mathrm{g}$ pHelper (Cell Biolabs Inc.). 48 hours after transfection, the medium was collected, pooled, and centrifuged for 15 minutes at $530 \mathrm{~g}$ to spin down floating cells. In parallel, cells were scraped and collected in PBS. Then, cells were lysed using a dry ice/ ethanol bath and centrifuged for 15 minutes at 3,350 $\mathrm{g}$ to discard cell debris. The cleared supernatant and the cleared medium were pooled and filtrated using a $0.22-\mu \mathrm{m}$ filter. The viral solution was filtrated through a cation-exchange membrane (Mustang S Acrodisc, Pall Corporation) to deplete empty particles and then filtrated through an anion-exchange membrane (Mustang Q Acrodisc, Pall Corporation) to retain AAV viral particles. Then, viruses were eluted and concentrated using Amicon Ultra-15 Centrifugal Filter Units. The usual titer was around $10^{11} \mathrm{PFU} / \mathrm{ml}$. For further details see Okada et al. (57).

\section{Virus injection in the sciatic nerve}

Mice were anesthetized with isoflurane inhalation and placed under a Stemi2000 microscope (Zeiss). The incision area was shaved and cleaned using betadine solution. After incision, the gluteus superficialis and biceps femoris muscles were separated to reveal the cavity traversed by the sciatic nerve. The nerve was lifted out using a spatula, and a thin glass needle filled with viral solution $(8 \mu \mathrm{l})$ was introduced into the nerve with a micromanipulator. This solution was injected over 30 minutes with short pressure pulses using a Picospritzer III (Parker Hannifin) coupled to a pulse generator. After injection, the nerve was placed back into the cavity, the muscles were readjusted, and the wound was closed using clips. For further details see Gonzalez et al. (19).

\section{Immunohistochemistry}

The dissected nerve was washed in L15 medium, fixed in Zamboni's fixative (58) for 10 minutes at room temperature, washed in PBS, and incubated in successive glycerol baths (15\%, 45\%, 60\%, 66\% glycerol in PBS) for 18 to 24 hours each, before freezing at $-20^{\circ} \mathrm{C}$. The nerves were cut in small pieces in $66 \%$ glycerol, and the perineurium sheet was removed. Small bundles of fibers were teased in double-distilled water on Superfrost slides and dried overnight at room temperature, and the slides were stored at $-20^{\circ} \mathrm{C}$. For immunostaining, the teased fibers were incubated for 1 hour at room temperature in blocking solution (10\% goat serum, $0.2 \%$ Triton X-100, and $0.01 \%$ sodium azide in PBS). Then, the samples were incubated with antiECCD2 primary mouse antibody (1:100; BD Biosciences, 610181), anti-phosphoS63-c-JUN primary mouse antibody (1:200; BD Biosciences, 558036), anti-c-JUN primary mouse antibody (1:200; BD Biosciences, 610326), anti-VDAC primary rabbit antibody (1:100; Cell Signaling, 4866), and/or MitoTracker Red (1:1,000; Molecular Probes, M7515) in blocking solution overnight at $4^{\circ} \mathrm{C}$. The next day, the samples were washed in PBS and incubated for 1 hour at room temperature with secondary donkey antibodies coupled to Alexa Fluor 488, Alexa Fluor 594, or Alexa Fluor 647 (1:1,600; Molecular Probes) and TO-PRO-3 iodide (50 $\mu \mathrm{M}$, Invitrogen, T3605). Finally, the samples were washed in PBS and mounted in Immu-Mount (Thermo Scientific). Images were acquired at room temperature using a $\times 20$ or $\times 40$ objective, a Zeiss confocal microscope (LSM710), and its associated software.

\section{Drug administration}

TRO19622 (Tocris Bioscience, 2906) was diluted in ethanol to $20 \mathrm{mM}$ and then diluted in sterile PBS to $2 \mu \mathrm{M}$. TRO19622 treatment was performed by intraperitoneal injection of $20 \mathrm{mM}$ of TRO19622 solution $(3 \mathrm{mg} / \mathrm{kg}$ ) or by injection into the sciatic nerve of $2 \mu \mathrm{l}$ of TRO19622 solution at $2 \mu \mathrm{M}$ using a Hamilton syringe or a glass needle in sterile conditions 30 minutes before multiphoton image acquisition or immunohistochemistry and demyelination studies, respectively. MJ (Sigma-Aldrich, 392707) was diluted in $1 \mathrm{ml}$ sterile PBS to $1 \mathrm{M}$ or $3 \mathrm{M}$. MJ treatment was performed by injection into the sciatic nerve of $1 \mu \mathrm{l}$ drug solution using a glass needle and a micromanipulator in sterile conditions, following the same virus injection procedure 2 hours before multiphoton image acquisition. Auranofin (Sigma-Aldrich, A6733) was diluted in DMSO to $10 \mathrm{mM}$ and then diluted in sterile PBS to $2 \mu \mathrm{M}$. Auranofin treatment was performed by injection into the sciatic nerve of $2 \mu$ l Auranofin solution using a Hamilton syringe during multiphoton image acquisition. CsA (Sigma-Aldrich, 30024-25MG) was diluted to $100 \mathrm{mM}$ in ethanol and then diluted in sterile PBS to $50 \mu \mathrm{M}$ and $500 \mu \mathrm{M}$. CsA treatment was performed by injection into the sciatic nerve of $4 \mu \mathrm{l}$ CsA solution using a glass needle and a microma- 


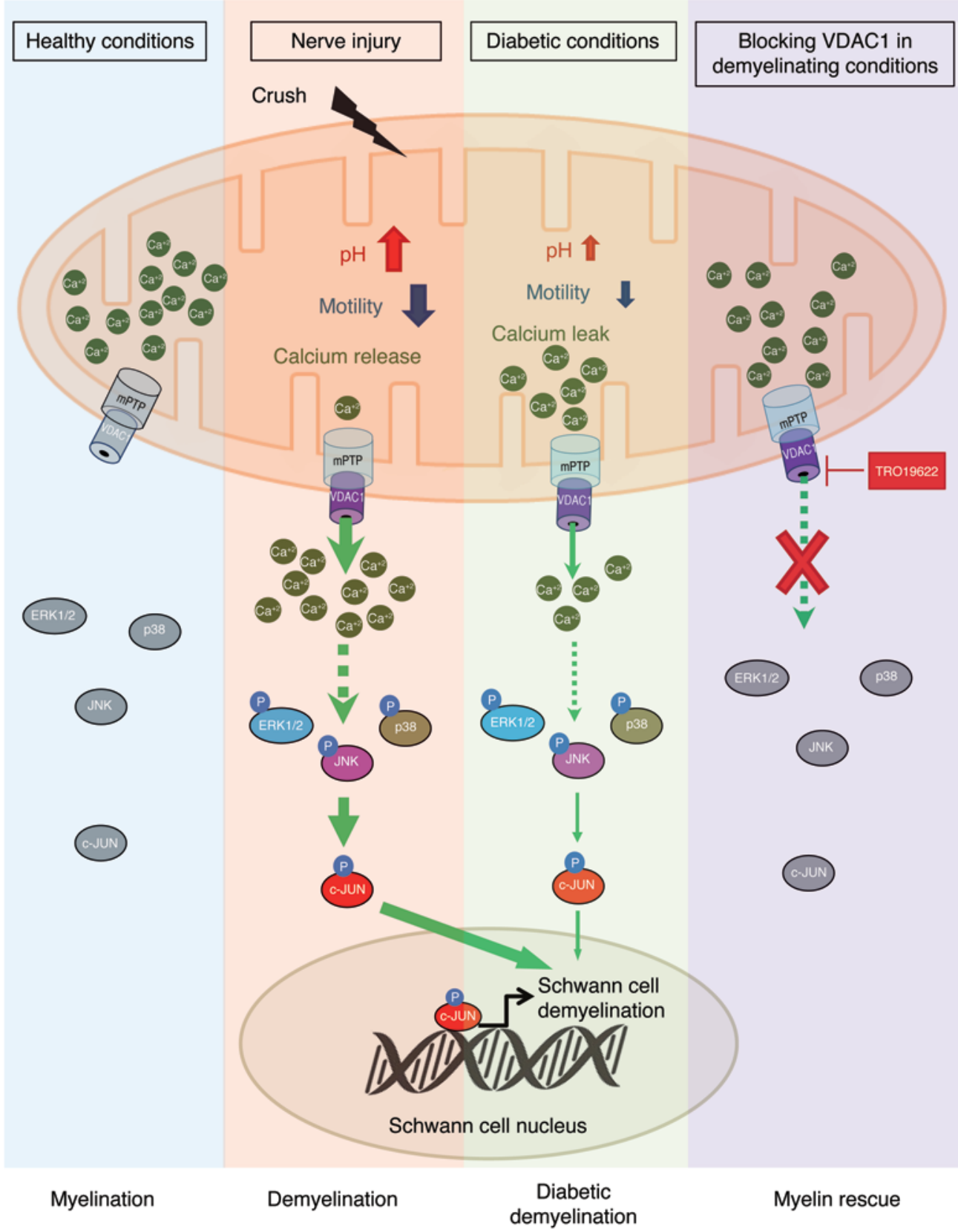

Figure 8. A graphical summary of the mitochondrial molecular mechanism of SC demyelination. After nerve injury (crush), mitochondria release calcium through VDAC1, leading to an increase of mitochondrial $\mathrm{pH}$ and a decrease of mitochondrial motility. MPTP formation is also required for the mitochondrial calcium release. This release of calcium induces phospho-ERK1/2, phospho-p38, phospho-JNK, and phospho-c-JUN activation, leading to SC demyelination. In diabetic conditions, this mechanism is altered and mitochondria leak calcium, which primes SCs for demyelination. However, blocking VDAC1-forming mPTP activity using TR019622 treatment induces inhibition of calcium release and the demyelination molecular pathway, allowing myelin sheath rescue.

nipulator in sterile conditions 30 minutes before multiphoton image acquisition. Ethanol was used as a vehicle for TRO19622, and PBS was used as a vehicle for MJ and CsA.

\section{Electron microscopy and morphometry}

Sciatic nerves of 24- and 32-week-old diabetic mice or 16- and 24-week-old CMT1A rats treated with TRO19622 or PBS were fixed for 20 minutes in situ with $4 \%$ PFA and $2.5 \%$ glutaraldehyde in $0.1 \mathrm{M}$ phosphate buffer ( $\mathrm{pH}$ 7.3). Then, nerves were removed and post-fixed overnight in the same buffer. After washing for 30 minutes in $0.2 \mathrm{M}$ PBS buffer, the nerves were incubated with $2 \%$ osmic acid in $0.1 \mathrm{M}$ phosphate buffer for 90 minutes at room temperature. Then, samples were washed in $0.2 \mathrm{M}$ PBS buffer, dehydrated using ethanol gradient solutions, and embedded in epoxy resin. Ultrathin (70-nm) cross sections were cut and stained with $1 \%$ uranyl acetate solution and lead citrate and analyzed using a HITACHI H7100 electron microscope at the Centre des Resources en Imagerie Cellulaire. For further details, see Cotter et al. (59). Semithin $(0.7-\mu \mathrm{m})$ cross sections were cut using a microtome (Leica, RM 2155) with a diamond knife (Histo HI 4317, Diatome). Sections were stained with toluidine blue (Sigma-Aldrich, 89640-5G) and observed using a light microscope (Leica Leitz DMRB). The g-ratio was determined using the ImageJ GRatioCalculator plug-in. At least 200 fibers per animal were analyzed.

\section{Multiphoton image acquisition}

All time-lapse images were obtained with a multiphoton microscope (Zeiss LSM 7 MP OPO). Mitochondria motility images were acquired by time-lapse recording of 1 image every 5 minutes during a 5-hour period at $920 \mathrm{~nm}$. GCaMP2 and SypHer probe images were acquired by time-lapse recording of 1 image every 15 minutes during a 5 -hour 
period at $985 \mathrm{~nm}$. Images were acquired at a constant laser intensity of $1 \%$ over a 100 -millisecond acquisition time, with $512 \times 512$ resolution and ten $20-\mu \mathrm{m} \mathrm{z}$-stack images. Images were saved in Zeiss.czi format and processed using the Image J program. For further details, see Gonzalez et al. (18).

\section{Electrophysiology}

Standard electroneurography was performed before, during, and after TRO19622 treatment on CMT1A rats and diabetic mice anesthetized with 3\% isoflurane. A pair of steel needle electrodes (AD Instruments, MLA1302) was placed subcutaneously along the nerve at the sciatic notch (proximal stimulation). A second pair of electrodes was placed along the tibial nerve above the ankle (distal stimulation). Supramaximal square-wave pulses, lasting $10 \mathrm{~ms}$ at $1 \mathrm{~mA}$ for mice and $3.6 \mathrm{~mA}$ for rats, were delivered using a PowerLab 26T (AD Instruments). CMAP was recorded from the intrinsic foot muscles using steel electrodes. Both amplitudes and latencies of CMAP were determined. The distance between the 2 sites of stimulation was measured alongside the skin surface with fully extended legs, and NCVs were calculated automatically from sciatic nerve latency measurements. For further details, see Fledrich et al. (43).

\section{Behavioral tests}

Rotarod test. A rotating rod apparatus (Bioseb) was used to measure neuromuscular coordination and balance of male diabetic mice at 20 weeks, and male and female CMT1A rats at 12 weeks, during vehicle or TRO19622 treatment. Mice and rats were first given a 2-day pretraining trial to familiarize them with the rotating rod. For control and diabetic mice, latency to fall was measured at a fixed speed of $10 \mathrm{rpm}$ over a 180-second time period. No acceleration testing was performed for mice. For control and CMT1A rats, latency to fall and maximum rpm were measured at accelerated speed. Rotating speed was successively increased from 4 to $40 \mathrm{rpm}$ in 10 -second intervals over a 300 -second time period. Each animal underwent 3 trials a day. For each day, values from the 3 trials were averaged for each animal, normalized according to animal weight, and then averaged for each treated group.

CatWalktest. Detailed analysis of gait was performed on free-walking 20-week-old diabetic mice and 12-week-old CMT1A rats using the CatWalk method (CatWalk XT 10.5, Noldus Information Technology) during vehicle or TRO19622 treatment. The runs were analyzed when the speed of crossing was constant and homogenous. At least 3 compliant runs were analyzed for each animal. Data were averaged for each animal, normalized according to animal weight, and then averaged for each treated group.

Grip test. Neuromuscular strength of diabetic mice and CMT1A rats was assessed in standardized grip strength tests for front limbs, hind limbs, and all limbs. All limbs grip strength was measured by supporting each rodent on a metal grid and pulling the animal's tail toward a horizontal grid connected to a gauge. Front and hind limb grip strengths were measured by supporting the forelimbs or hind limbs, respectively, and pulling the animal's tail toward a horizontal T-bar connected to a gauge. The maximum force (measured in newtons) exerted on the T-bar or grid before the animal lost its grip was recorded, and the mean of 3 repeated measurements was calculated. All data were normalized according to animal weight.

\section{Statistics}

Data are represented as mean \pm SD or mean \pm SEM. Statistical significance was determined using a 2-tailed Student's $t$ test or 1-way ANOVA, followed by a Dunnett's multiple comparison post-hoc test. A $P$ value of less than 0.05 was considered significant. In figure legends, $n$ represents the number of independent experiments.

\section{Study approval}

All animal experiments were approved by the Comité d'Ethique en Expérimentation Animale du Languedoc-Roussillon, Montpellier, France, and the Ministère de l'Enseignement Supérieur et de la Recherche, Paris, France (CEEA-LR approval 11032).

\section{Author contributions}

SG and NT wrote the paper. SG, JB, CPT, JJ, and RF performed experiments. SG, RC, GL, and NT analyzed data. GL provided expertise in mitochondrial biology. NT supervised the project.

\section{Acknowledgments}

We acknowledge $\mathrm{H}$. Boukhadaoui (Montpellier RIO Imaging Platform), C. Cazevieille and A. Caballero-Megido (Centre des Resources en Imagerie Cellulaire - TEM platform), and the Animal Facility of the Institute for Neurosciences of Montpellier for help and technical assistance. We would like to thank J.C. Jonas (Université Catholique de Louvain, Belgium) for the mito-SypHer probe, X. Wang (Peking University, China) for the mito-GCaMP2 probe, M. Sereda (Göttingen, Germany) for the CMT1A rat model, A.L. Bonnefont (Montpellier, France) for behavior tests assistance, W. Joly for critical reading of the manuscript, and P. Bomont, M. Teigell, and C. Raoul for technical help. This work was supported by European Research Council grant (FP7-IDEAS-ERC 311610 to N. Tricaud), an INSERM-AFM Avenir grant (to N. Tricaud), and the StratNeuro program (to R. Chrast).

Address correspondence to: Nicolas Tricaud, Institute for Neurosciences of Montpellier, INSERM U1051, 80 rue Augustin Fliche, 34091, Montpellier, France. Phone: 33.0.4.99.63.61.40; E-mail: nicolas.tricaud@inserm.fr.
1. Nave KA. Myelination and support of axonal integrity by glia. Nature. 2010;468(7321):244-252.

2. Sherman DL, Brophy PJ. Mechanisms of axon ensheathment and myelin growth. Nat Rev Neurosci. 2005;6(9):683-690.

3. Hughes RA. Peripheral neuropathy. BMJ. 2002;324(7335):466-469.

4. Zochodne DW. Diabetic polyneuropathy: an update. Curr Opin Neurol. 2008;21(5):527-533.
5. Zenker J, Ziegler D, Chrast R. Novel pathogenic pathways in diabetic neuropathy. Trends Neurosci. 2013;36(8):439-449.

6. Pham K, Gupta R. Understanding the mechanisms of entrapment neuropathies. Review article. Neurosurg Focus. 2009;26(2):E7.

7. Harati Y. Diabetic peripheral neuropathies. Ann Intern Med. 1987;107(4):546-559.

8. Sugimoto K, Murakawa Y, Sima AA. Diabetic neu- ropathy - a continuing enigma. Diabetes Metab Rev. 2000;16(6):408-433.

9. Malik RA. Pathology of human diabetic neuropathy. Handb Clin Neurol. 2014;126:249-259.

10. Skre H. Genetic and clinical aspects of Charcot-Marie-Tooth's disease. Clin Genet. 1974;6(2):98-118.

11. Timmerman V1, Strickland AV2, Züchner S3 Genetics of Charcot-Marie-Tooth (CMT) disease 
within the frame of the human genome project success. Genes (Basel). 2014;5(1):13-32.

12. Lupski JR, et al. DNA duplication associated with Charcot-Marie-Tooth disease type 1A. Cell. 1991;66(2):219-232.

13. Chittoor-Vinod VG, Lee S, Judge SM, Notterpek L. Inducible HSP7O is critical in preventing the aggregation and enhancing the processing of PMP22. ASN Neuro. 2015;7(1):1759091415569909.

14. Viader A, et al. Aberrant Schwann cell lipid metabolism linked to mitochondrial deficits leads to axon degeneration and neuropathy. Neuron. 2013;77(5):886-898.

15. Fünfschilling U, et al. Glycolytic oligodendrocytes maintain myelin and long-term axonal integrity. Nature. 2012;485(7399):517-521.

16. Viader A, Golden JP, Baloh RH, Schmidt RE, Hunter DA, Milbrandt J. Schwann cell mitochondrial metabolism supports long-term axonal survival and peripheral nerve function. J Neurosci. 2011;31(28):10128-10140.

17. Fernyhough P, Roy Chowdhury SK, Schmidt RE. Mitochondrial stress and the pathogenesis of diabetic neuropathy. Expert Rev Endocrinol Metab. 2010;5(1):39-49.

18. Gonzalez S, et al. In vivo time-lapse imaging of mitochondria in healthy and diseases peripheral myelin sheath. Mitochondrion. 2015;23:32-41.

19. Gonzalez S, et al. In vivo introduction of transgenes into mouse sciatic nerve cells in situ using viral vectors. Nat Protoc. 2014;9(5):1160-1169.

20. Chen M, Wang Y, Hou T, Zhang H, Qu A, Wang $\mathrm{X}$. Differential mitochondrial calcium responses in different cell types detected with a mitochondrial calcium fluorescent indicator, mitoGCaMP2. Acta Biochim Biophys Sin (Shanghai). 2011;43(10):822-830.

21. Roma LP, Duprez J, Takahashi HK, Gilon P, Wiederkehr A, Jonas JC. Dynamic measurements of mitochondrial hydrogen peroxide concentration and glutathione redox state in rat pancreatic $\beta$-cells using ratiometric fluorescent proteins: confounding effects of $\mathrm{pH}$ with HyPer but not roGFP1. Biochem J. 2012;441(3):971-978.

22. Shoshan-Barmatz V, Gincel D. The voltagedependent anion channel: characterization, modulation, and role in mitochondrial function in cell life and death. Cell Biochem Biophys. 2003;39(3):279-292.

23. Ritchie JM. Voltage-gated cation and anion channels in mammalian Schwann cells and astrocytes. Physiol (Paris). 1987;82(4):248-257.

24. Clapham DE. Calcium signaling. Cell. 2007;131(6):1047-1058.

25. Shoshan-Barmatz V, Israelson A, Brdiczka D, Sheu SS. The voltage-dependent anion channel (VDAC): function in intracellular signalling, cell life and cell death. Curr Pharm Des. 2006;12(18):2249-2270.

26. Bordet $\mathrm{T}$, et al. Identification and charac- terization of cholest-4-en-3-one, oxime (TRO19622), a novel drug candidate for amyotrophic lateral sclerosis.J Pharmacol Exp Ther. 2007;322(2):709-720.

27. Goldin N, et al. Methyl jasmonate binds to and detaches mitochondria-bound hexokinase. Oncogene. 2008;27(34):4636-4643.

28. Bernardi P. The mitochondrial permeability transition pore: a mystery solved? Front Physiol. 2013;4:95.

29. Sharov VG, Todor A, Khanal S, Imai M, Sabbah HN. Cyclosporine A attenuates mitochondrial permeability transition and improves mitochondrial respiratory function in cardiomyocytes isolated from dogs with heart failure. J Mol Cell Cardiol. 2007;42(1):150-158.

30. Rigobello MP, Scutari G, Boscolo R, Bindoli A. Induction of mitochondrial permeability transition by auranofin, a gold(I)-phosphine derivative. Br J Pharmacol. 2002;136(8):1162-1168.

31. Gainutdinov T, et al. Knockout of cyclophilin D in Ppif $^{\prime-}$ mice increases stability of brain mitochondria against $\mathrm{Ca}^{2+}$ stress. Arch Biochem Biophys. 2015;579:40-46.

32. Baines CP, et al. Loss of cyclophilin D reveals a critical role for mitochondrial permeability transition in cell death. Nature. 2005;434(7033):658-662.

33. Jessen KR, Mirsky R. The origin and development of glial cells in peripheral nerves. Nat Rev Neurosci. 2005;6(9):671-682.

34. Salzer JL. Switching myelination on and off. JCell Biol. 2008;181(4):575-577.

35. Feldman EL. Oxidative stress and diabetic neuropathy: a new understanding of an old problem. JClin Invest. 2003;111(4):431-433.

36. Kolb H. Mouse models of insulin dependent diabetes: low-dose streptozocin-induced diabetes and nonobese diabetic (NOD) mice. Diabetes Metab Rev. 1987;3(3):751-778.

37. Hamilton RT, et al. Elevated protein carbonylation, and misfolding in sciatic nerve from $\mathrm{db} /$ $\mathrm{db}$ and Sod1(-/-) mice: plausible link between oxidative stress and demyelination. PLoS One. 2013;8(6):e65725.

38. Wang L, et al. Phosphodiesterase- 5 is a therapeutic target for peripheral neuropathy in diabetic mice. Neuroscience. 2011;193:399-410.

39. Robertson DM, Sima AA. Diabetic neuropathy in the mutant mouse [C57BL/ks(db/db)]: a morphometric study. Diabetes. 1980;29(1):60-67.

40. Fazan SVP, De Vasconcelos CCA, Valença MM, Nessler R, Moore KC. Diabetic peripheral neuropathies: a morphometric overview. Int J Morphol. 2010;28(1):51-64.

41. Zenker J, at al. Altered distribution of juxtaparanodal kv1.2 subunits mediates peripheral nerve hyperexcitability in type 2 diabetes mellitus. J Neurosci. 2012;32(22):7493-7498.

42. Sereda $\mathrm{M}$, et al. A transgenic rat model of Charcot-Marie-Tooth disease. Neuron. 1996;16(5):1049-1060.

43. Fledrich R, et al. Soluble neuregulin-1 modu- lates disease pathogenesis in rodent models of Charcot-Marie-Tooth disease 1A. Nat Med. 2014;20(9):1055-1061.

44. Murphy SM, et al. Charcot-Marie-Tooth disease: frequency of genetic subtypes and guidelines for genetic testing. J Neurol Neurosurg Psychiatry. 2012;83(7):706e710.

45. Lee HJ, Shin YK, Park HT. Mitogen activated protein kinase family proteins and c-jun signaling in injury-induced Schwann cell plasticity. Exp Neurobiol. 2014;23(2):130-137.

46. Parkinson DB, et al. c-Jun is a negative regulator of myelination. J Cell Biol. 2008;181(4):625-637.

47. Hantke J, et al. c-Jun activation in Schwann cells protects against loss of sensory axons in inherited neuropathy. Brain. 2014;137(pt 11):2922-2937.

48. De Pinto V, Messina A, Lane DJ, Lawen A. Voltage-dependent anion-selective channel (VDAC) in the plasma membrane. FEBS Lett. 2010;584(9):1793-1799.

49. Chuderland D, Seger R. Calcium regulates ERK signaling by modulating its protein-protein interactions. Commun Integr Biol. 2008;1(1):4-5.

50. Enslen H, Tokumitsu H, Stork PJ, Davis RJ, Soderling TR. Regulation of mitogen-activated protein kinases by a calcium/calmodulin-dependent protein kinase cascade. Proc Natl Acad Sci U S A. 1996;93(20):10803-10808.

51. Javadov S, Jang S, Agostini B. Crosstalk between mitogen-activated protein kinases and mitochondria in cardiac diseases: therapeutic perspectives. Pharmacol Ther. 2014;144(2):202-225.

52. Hantke J, et al. A mutation in an alternative untranslated exon of hexokinase 1 associated with hereditary motor and sensory neuropathy - Russe (HMSNR). Eur J Hum Genet. 2009;17(12):1606-1614.

53. Schwartz MW, Baskin DG. Leptin and the brain: then and now. JClin Invest. 2013;123(6):2344-2345.

54. Martin LJ. Olesoxime, a cholesterol-like neuroprotectant for the potential treatment of amyotrophic lateral sclerosis. IDrugs. 2010;13(8):568-580.

55. Lobsiger CS, et al. Schwann cells expressing dismutase active mutant SOD1 unexpectedly slow disease progression in ALS mice. Proc Natl Acad Sci U S A. 2009;106(11):4465-4470.

56. He TC, Zhou S, da Costa LT, Yu J, Kinzler KW, Vogelstein B. A simplified system for generating recombinant adenoviruses. Proc Natl Acad Sci U S A. 1998;95(5):2509-2514.

57. Okada T, et al. Scalable purification of adenoassociated virus serotype 1 (AAV1) and AAV8 vectors, using dual ion-exchange adsorptive membranes. Hum Gene Ther. 2009;20(9):1013-1021.

58. Stefanini M, De Martino C, Zamboni L. Fixation of ejaculated spermatozoa for electron microscopy. Nature. 1967;216(5111):173-174.

59. Cotter L, et al. Dlg1-PTEN interaction regulates myelin thickness to prevent damaging peripheral nerve overmyelination. Science. 2010;328(5984):1415-1418. 